\title{
Combined Sector and Channel Hopping Schemes for Efficient Rendezvous in Directional Antenna Cognitive Radio Networks
}

\author{
AbdulMajid M. Al-Mqdashi, ${ }^{1}$ A. Sali, ${ }^{1}$ Nor K. Noordin, ${ }^{1}$ \\ Shaiful J. Hashim, ${ }^{1}$ and Rosdiadee Nordin ${ }^{2}$ \\ ${ }^{1}$ Wireless and Photonic Networks Research Centre of Excellence (WiPNEt), Department of Computer and \\ Communication Systems Engineering, Faculty of Engineering, Universiti Putra Malaysia, 43400 Serdang, Selangor, Malaysia \\ ${ }^{2}$ Department of Electrical, Electronic, and Systems Engineering, Universiti Kebangsaan Malaysia, 43600 Bangi, Selangor, Malaysia
}

Correspondence should be addressed to AbdulMajid M. Al-Mqdashi; abdulmajid.almqdashi@gmail.com

Received 27 August 2017; Accepted 24 October 2017; Published 12 December 2017

Academic Editor: Ahmed M. Al-Samman

Copyright (C) 2017 AbdulMajid M. Al-Mqdashi et al. This is an open access article distributed under the Creative Commons Attribution License, which permits unrestricted use, distribution, and reproduction in any medium, provided the original work is properly cited.

\begin{abstract}
Rendezvous is a prerequisite and important process for secondary users (SUs) to establish data communications in cognitive radio networks (CRNs). Recently, there has been a proliferation of different channel hopping- (CH-) based schemes that can provide rendezvous without relying on any predetermined common control channel. However, the existing $\mathrm{CH}$ schemes were designed with omnidirectional antennas which can degrade their rendezvous performance when applied in CRNs that are highly crowded with primary users (PUs). In such networks, the large number of PUs may lead to the inexistence of any common available channel between neighboring SUs which result in a failure of their rendezvous process. In this paper, we consider the utilization of directional antennas in CRNs for tackling the issue. Firstly, we propose two coprimality-based sector hopping (SH) schemes that can provide efficient pairwise sector rendezvous in directional antenna CRNs (DIR-CRNs). Then, we propose an efficient $\mathrm{CH}$ scheme that can be combined within the SH schemes for providing a simultaneous sector and channel rendezvous. The guaranteed rendezvous of our schemes are proven by deriving the theoretical upper bounds of their rendezvous delay metrics. Furthermore, extensive simulation comparisons with other related rendezvous schemes are conducted to illustrate the significant outperformance of our schemes.
\end{abstract}

\section{Introduction}

The inefficiency of radio spectrum utilization due to the fixed assignment of spectrum bands, coupled with the huge increase in the number of wireless devices recently, leads to the emerging of cognitive radios (CRs). CRs have been introduced as an efficient solution for the spectrum scarcity issue. They allow unlicensed users, also known as secondary users (SUs), to opportunistically use licensed bands as long as this use does not create any interference to the bands licensed users, that is, primary users (PUs). In distributed CRNs, SUs need to meet each other on a common channel and exchange control messages in order to set up their data transmissions [1]. This operation is called rendezvous, which is a fundamental and a vital process for initiating the connection of the SUs data communications.
The use of a dedicated common control channel (CCC) is one widely adopted rendezvous approach in the literature [2-6]. Although this approach can simplify the rendezvous process, it has many drawbacks such as the CCC susceptibility to long-time blocking by PUs, early saturation by SUs, or jamming by attackers $[7,8]$. Moreover, the spectrum heterogeneity among SUs, caused by the spatial and temporal variations of the spectrum opportunities (influenced by neighboring PU activities), makes this approach not practical. Therefore, channel hopping $(\mathrm{CH})$ has been proposed as an alternative approach for rendezvous without the need for any predefined CCC. The existing $\mathrm{CH}$-based rendezvous schemes were designed with omnidirectional antenna according to different mathematical structures (e.g., [9-18]) in order to provide a successful channel rendezvous between a pair of single-hop SUs. In the $\mathrm{CH}$ approach, each $\mathrm{SU}$ generates its $\mathrm{CH}$ sequence 
based on the available channels that are sensed to be idle from any PU activities within its omnidirectional range. Then, the SU keeps hopping over the channels according to the generated $\mathrm{CH}$ sequence for achieving channel rendezvous. The rendezvous occurs between a pair of communicating SUs when they hop during the same time slot over a channel that is commonly available for both of them. The existence of common available channels (at least one) between the pair of communicating SUs is a common and essential assumption made by all the existing $\mathrm{CH}$ schemes. Their designs rely mainly on this assumption to take place for ensuring the success of the rendezvous operation. However, none of the existing $\mathrm{CH}$ rendezvous designs were tailored for highdensity PU networks, where the number of active PUs in the network is larger than the number of the channels. In such networks, the channel availabilities may vary dramatically among the SUs within the single-hop rendezvous region itself. This can lead to the inexistence of any common available channel between a pair of SUs and hence the failure of the rendezvous process.

One approach to overcome this serious rendezvous problem is using directional antennas instead of the conventionally used omnidirectional ones [19]. Directional antennas have been utilized in emerging wireless networks such as the millimeter-wave (MMW) networks for providing highly reliable transmission and multi-Gigabit data rates. This is mainly due to their capabilities in enlarging the transmission range and limiting the interference $[20,21]$. However, In CRNs, equipping SUs with directional antennas for channel rendezvous can bring about many advantages. First of all, it allows SUs to transmit their rendezvous messages towards specific directions which can reduce the amount of interference to the PUs in the network as compared with the omnidirectional antennas. This is because the transmission of the SU that is equipped with directional antenna is directed to a particular region which can only cause interference to those PUs that are within this region. On the contrary, when using the omnidirectional antennas, the transmission of the SU is scattered towards all the directions. Thus, it can cause interference to all the surrounding PUs within the SU transmission range. Second, as related to the interference restriction imposed by the CR concept, SUs can use only the channels that are idle from any PU activities within their transmission range. According to that and due to its directed transmission range, using directional antenna will increase the number of available channels that can be utilized by the SU in each of its transmission sectors. As a consequence, the probability for any pair of neighbouring SUs to have at least a common available channel within their crossed sectors is high regardless of the number of PUs in the CRN. Note that, in omnidirectional antennas, this probability could be less than 0.1 for some scenarios when the number of PUs is larger than the network channels [19]. Increasing the probability of existing common available channel between the SUs will significantly enhance the probability of successful channel rendezvous. Therefore, in this paper, we study the rendezvous problem in directional antenna CRNs where SUs are equipped with directional antennas.
While the use of directional antennas for channel rendezvous in CRNs can bring about the above-mentioned advantages, it also imposes some unique challenges. Among these challenges is the sector rendezvous which must be achieved in advance between the pair of communicating SUs before they can achieve channel rendezvous. Specifically, the SUs need to steer their antennas towards each other in order to communicate over their commonly available channels. This necessitates the design of a deterministic sector hopping (SH) scheme that can guarantee that any pair of neighbouring SUs will steer their antennas to the proper directions (i.e., towards each other) at a certain time instance. So, when the $\mathrm{SH}$ scheme is combined with a suitable and deterministic $\mathrm{CH}$ scheme, SUs can achieve successful sector and channel rendezvous simultaneously within a bounded time. However, since the SUs do not know the relative locations of each other as well as the available channels before they rendezvous, the combined sector and channel hopping (SCH) scheme should be designed in fully distributed manner without any prior information or synchronization. Designing such combined $\mathrm{SCH}$ schemes for achieving rendezvous in DIR-CRNs is intuitively more challenging as compared with the traditional omnidirectional antenna paradigm.

In spite of the existing research in the literature, there have been some papers that addressed the utilization of directional antennas in CRNs. However, most of the proposed works investigated issues other than the rendezvous issue such as sensing $[22,23]$, routing $[24,25]$, and connectivity $[26,27]$. To the best of our knowledge, the works in $[19,28]$ are the only proposals that tackle the sector and channel rendezvous issue in DIR-CRNS. In [19], an efficient framework for beamforming was proposed to provide a pairwise sector and channel rendezvous in DIR-CRNs. However, the SH scheme in [19] is not a blind design, where it assumes that the receiver must have prior knowledge of the sender number of sectors in order to achieve a successful sector rendezvous. This is not practical, since SUs do not know any information about each other before they rendezvous. On the other hand, $\mathrm{Li}$ and Xie in [28] proposed a blind prime-based SH scheme, where the number of sectors is adjusted to primes for achieving a guaranteed sector rendezvous. However, since the scheme relies on the coprimality between prime numbers only, the authors added an initiating rotated $\mathrm{SH}$ sequence on the sender side. This is in order to ensure a successful rendezvous with the receiver when the sender and the receiver construct their sequences with the same prime. Accordingly, the extra sequence overhead incurs extensively long sector rendezvous latency and hence a long time-to-rendezvous (TTR) will result when the $\mathrm{SH}$ is combined with a $\mathrm{CH}$ sequence. Furthermore, the SH schemes in $[19,28]$ are designed only for asymmetric-role environment, where SUs have preassigned role (i.e., SU is either a sender or receiver). However, this design limits the applications of the schemes; for example, SUs cannot work as a forwarder (i.e., receive packets from one SU and then forward them to another SU) due to the permanent role assignment [29]. Another drawback of these works is their limitations to provide a complete solution for combining the SH scheme with a suitable and efficient $\mathrm{CH}$ scheme which consider the unique traits of DIR-CRNs. 
They claimed that their $\mathrm{SH}$ schemes can work on top of any existing deterministic $\mathrm{CH}$ schemes which were designed for the omnidirectional antennas paradigm. However, the combined $\mathrm{CH}$ scheme must be designed appropriately by taking into account the unique traits of DIR-CRNs such as the channels' qualities in the different sectors.

In this paper, we propose efficient schemes for achieving rendezvous in DIR-CRNs. The main contributions of this paper are summarized as follows:

(i) We propose two asymmetric- and symmetric-role coprimality-based SH schemes, called PES-SH and IPES-SH, for pairwise sector rendezvous in DIRCRNs.

(ii) We combined our SH schemes with an efficient gridquorum-based $\mathrm{CH}$ scheme, where the $\mathrm{CH}$ sequences are generated based on the best-quality channels in the sectors. The combined SCH schemes will provide guaranteed sector and channel rendezvous between the pair of SUs.

(iii) We derive the upper bound of the rendezvous delay metrics for all of our schemes. Also, we conduct extensive simulations to study their performance under various network settings and compare them with the related existing works in $[19,28,30]$.

The rest of this paper is organized as follows. The system model and problem formulation are presented in Section 2. The design and theoretical analysis of the proposed $\mathrm{SH}$ schemes are presented in Sections 3 and 4. In Section 5, we present the combined sector and channel hopping schemes. Using simulations, in Section 6, we evaluate the performance of our proposed schemes and compare their performance with other comparable schemes. Finally, we conclude the paper in Section 7.

\section{Models and Problem Definition}

In this section, we present the system model and rendezvous problem definition in DIR-CRNs.

2.1. System Model. We consider a CRN consisting of $K$ SUs that coexist with several PUs in an $w \times w$ area. There are totally $L$ primary channels which can be accessed opportunistically by the SUs in order to communicate with each other. Each SU $i \in K$ is equipped with a directional antenna with beamwidth $\theta_{i}\left(0<\theta_{i}<2 \pi\right)$. Accordingly, the $2 \pi$ communication range of the SU $i$ is divided into $N_{i}=\left(2 \pi / \theta_{i}\right)$ nonoverlapping sectors that are indexed from 1 to $N_{i}$. However, the number of sectors and the orientation of the sector indexing (i.e., either clockwise or anticlockwise) by each SU may be different from the others (see Figure 1). This is a very practical issue because each SU will configure its sectors based only on its own view to the surrounding environment. Each SU can transmit over any of its transmission sectors as long as it does not make any interference to any active PU transmission. Accordingly, we assume that the transmission sectors are also used as sensing sectors by which every SU can sense the appearance of the active PUs within them. Thus, the SU can obtain the channel availability information per each sector. We consider a timeslotted communication, where time is divided into discrete slots that have fixed and equal durations. During each time slot, we assume that a SU can only transmit in one sector over a single channel.

2.2. Definitions of Sector and Channel Rendezvous. In this paper, we mainly focus on the pairwise sector and channel rendezvous between any pair of SUs in a DIR-CRN. Firstly, we define the sector rendezvous problem as follows.

2.2.1. The Sector Rendezvous Problem. For any two neighbour SUs (say $\mathrm{SU}_{i}$ and $\mathrm{SU}_{j}$ ) that are equipped with directional antennas and want to communicate with each other, assume that $\mathrm{SU}_{i}$ is located in the sector $h_{j} \in\left[1, N_{j}\right]$ of $\mathrm{SU}_{j}$ and $\mathrm{SU}_{j}$ is situated in the sector $h_{i} \in\left[1, N_{i}\right]$ of $\mathrm{SU}_{i}$. The pair of the sectors $\left(h_{i}, h_{j}\right)$ is called the sector rendezvous pair. SUs are said to achieve a successful sector rendezvous if and only if they steer their antennas towards each other, where their transmission sectors can cover each other. Formally, let $S_{i}=$ $\left\{S_{i}^{0}, S_{i}^{1}, \ldots, S_{i}^{T_{i}}\right\}$ and $S_{j}=\left\{S_{j}^{0}, S_{j}^{1}, \ldots, S_{j}^{T_{j}}\right\}$ denote the sector hopping sequences for $\mathrm{SU}_{i}$ and $\mathrm{SU}_{j}$, with periods $T_{i}$ and $T_{j}$, respectively. Also let $\delta$ denote the clock drift between $\mathrm{SU}_{i}$ and $\mathrm{SU}_{j}$ in the asynchronous scenario. The sector rendezvous problem can be formulated as follows:

$$
\begin{aligned}
& \text { If } \forall \delta, \forall N_{i}, N_{j}, \exists t \in\left[0, T_{i} T_{j}-1\right] \text {, s.t, }\left(S_{i}^{t}=h_{i}\right. \text { and } \\
& \left.S_{j}^{t+\delta}=h_{j}\right) \text {, then the sector rendezvous is achieved } \\
& \text { over the sector rendezvous pair }\left(h_{i}, h_{j}\right) .
\end{aligned}
$$

2.2.2. The Sector and Channel Rendezvous Problem. When a SU steers its directional antenna to each sector of its transmission sectors, it performs $\mathrm{CH}$ over the available channels within the sector according to a $\mathrm{CH}$ sequence. This is in order to attempt channel rendezvous with another SU over a commonly available channel between them. The combined $\mathrm{CH}$ scheme should be designed in such a way that guarantees a successful channel rendezvous within bounded time whenever the communicating SUs already achieved a successful sector rendezvous. During the current sector, if the SU does not achieve channel rendezvous with its intended communicating SU within the bounded time slots, it then steers its antenna to the next sector of its $\mathrm{SH}$ sequence and executes the $\mathrm{CH}$ scheme based on the available channels within this sector.

One approach to view the problem is to look at it in a hierarchical way, in which time is divided into frames, where the SU tunes its antenna to one sector during each frame. In other words, the $\mathrm{SH}$ is implemented on the frame scale. Then, each frame is divided into number of time slots, where the combined $\mathrm{CH}$ sequence is executed (i.e., the $\mathrm{CH}$ is implemented on the time slot scale). The main goal of this paper is to design deterministic combined SCH scheme so as to tackle the sector and channel rendezvous problem.

2.2.3. Metrics. The proposed $\mathrm{SH}$ schemes as well as the combined SCH schemes will be evaluated according to the following metrics: 


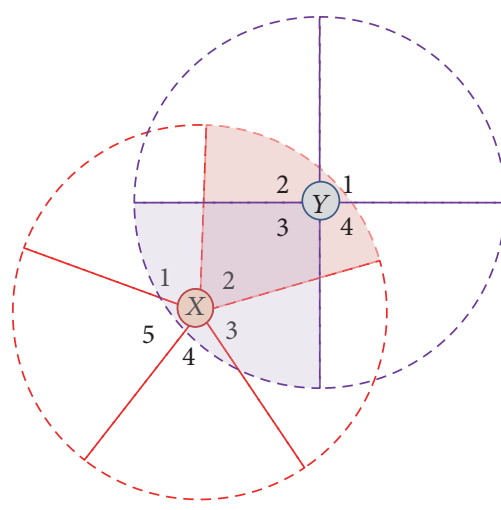

(a) Pair of $X$ and $Y$ SUs

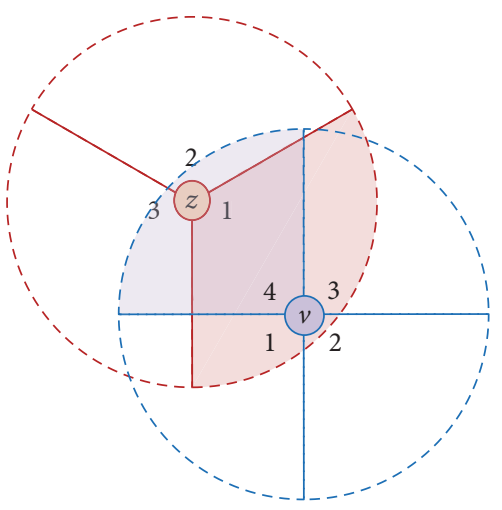

(b) Pair of $V$ and $Z$ SUs

FIGURE 1: Examples of directional antenna configurations in two pairs of communicating SUs: (a) $\mathrm{SU}_{X}$ and $\mathrm{SU}_{Y}$ divide their transmission range into $N_{X}=5$ and $N_{Y}=4$ sectors; the rendezvous sector pair is $(2,3)$. (b) $\mathrm{SU}_{V}$ and $\mathrm{SU}_{Z}$ divide range into $N_{v}=4$ and $N_{z}=3$ sectors; the rendezvous sector pair is $(4,1)$.

(i) Sector rendezvous latency (SRL): it is defined as the required latency (in number of sector frames) for a pair of SUs to achieve sector rendezvous. We consider the maximum and average latency (MSRL/ASRL). MSRL is defined as the upper bound of the SRL between the SUs for all the possible clock drifts between them

(ii) Time-to-rendezvous (TTR): it is defined as the required time (in number of time slots) for a pair of SUs to achieve sector and channel rendezvous simultaneously. Maximum and average time-to-rendezvous (MTTR/ATTR) are considered. MTTR means the required time for a guaranteed rendezvous even in the worst case.

\section{Prime- and Even-Based Sequences Sector Hopping (PES-SH) Scheme}

In this section, we propose an asymmetric-role $\mathrm{SH}$ scheme, called prime- and even-based sequences sector hopping (PES-SH) scheme, and then we analyze its theoretical performance.

3.1. Scheme Design. For ensuring a successful sector rendezvous between a pair of communicating SUs, the key for the deterministic design of our PES-SH scheme is to construct two $\mathrm{SH}$ sequences based on two coprime numbers. However, the coprimality is maintained between a pair of prime and even numbers that are relatively prime (i.e., coprime). In our PES-SH scheme, we assume that $N_{\max }$ is a global variable known by all SUs, which indicates the maximum number of sectors by which any SU is allowed to divide its omnidirectional $2 \pi$ transmission range. The value of $N_{\max }$ is obtained based on the number of channels in the network; for example, for $L$ number of channels, the value of $N_{\max }$ could be $2 \times L$. Accordingly, we consider two avoided sets:

(1) The Avoided Prime Set (APS) which contains the primes $\{2,3\}$
(2) The Avoided Even Set (AES) which contains any even number in the range $\left[2, P_{\max }\right]$, such that this even number is dividable by any prime number in the range $\left[5, P_{\max }\right] . P_{\max }$ is the smallest prime not less than $N_{\max }$. For example, if $N_{\max }=20$, then $P_{\max }=23$ and hence AES $=\{10,14,20,22\}$

The APS and AES sets are avoided by the sender and receiver, respectively, when they construct their $\mathrm{SH}$ sequences. Without loss of generality, assume that sender and receiver have $N_{s}$ and $N_{r}$ antenna sectors, respectively, where $N_{s}, N_{r} \in$ $\left[1, N_{\max }\right]$. The main idea for the rendezvous of our PES-SH scheme is to let one SU (sender) adjust the number of its sectors to be the smallest prime number greater than $N_{s}$. On the other hand, the other SU (receiver) adjusts the number of its sectors to the smallest even number which is not smaller than $N_{r}$ and not in the AES. Accordingly, the adjusted prime number of sectors for the sender and the adjusted even number of sectors for the receiver are guaranteed to be relatively prime.

Algorithms 1 and 2 are used by the sender and receiver, respectively, to construct each round of their $\mathrm{SH}$ sequences. The generating steps of the PES-SH sequences are as follows.

3.1.1. Sender Sequence in PES-SH. When a SU has data to transmit, it serves as a sender and hence generates a primebased (PS-SH) sequence as follows. First, given the sector set $S=\left\{1, \ldots, N_{s}\right\}$, randomly select a starting sector index $i \in\left[1, N_{s}\right]$ and rotate $S$ circularly starting from $i$ as $S=$ rotate $(S, i-1)$. Second, adjust $N_{s}$ to be the smallest prime number $P_{s}$ which is not smaller than $N_{s}$ and is not in the APS set (i.e., $P_{s}$ should be 5 when $N_{s}<5$ ). Third, construct each round of the PS sequence which has a length equal to $P_{S}$ as follows: the $k_{\text {th }}$ sector index of the PS round is $S(k)$ if $k \leq N_{s}$; otherwise the $k_{\text {th }}$ sector is selected randomly form $S$ when $k>N_{s}$. The sender will keep steering its antenna according to the PS sequence until it achieves sector rendezvous with its intended receiver.

Consider the cases for the sender SUs $X$ and $V$ in Figure 1; SU $X$ in Figure 1(a) has a prime number of sectors $N_{x}=5$, 
Input: $N_{s}, S_{s}$

Output: a round $\omega$ of the PS sequence for the sender $s$.

(1) Find the smallest prime $\left(P_{s}>3\right)$ that is not smaller than $N_{s}$.

(2) for $t=0: P_{s}-1$ do

(3) if $\left(\left(t \bmod P_{s}\right)<N_{s}\right)$ then

(4) $\quad \omega_{s}(t)=S_{s}(t+1)$.

(5) else

(6) $\quad r$ is a random number $\in\left[1, N_{s}\right]$

(7) $\quad \omega_{s}(t)=S_{s}(r)$

(8) end if

(9) end for

(10) return $\omega_{s}$.

Algorithm 1: The sender SH generation algorithm (PS-SH).
Input: $N_{r}, S_{r}$, AES.

Output: a round $\gamma$ of the ES-SH sequence for the receiver $r$.

(1) Find $\left(E_{r}: E_{r} \notin \mathrm{AES}\right)$ as the smallest even number that is not smaller than $N_{r}$.

(2) for $t=0: E_{r}-1$ do

(3) if $\left(\left(t \bmod E_{r}\right)<N_{r}\right)$ then

(4) $\gamma_{r}(t)=S_{r}(t+1)$.

(5) else

(6) $\quad r$ is a random number $\in\left[1, N_{r}\right]$

(7) $\quad \gamma_{r}(t)=S_{r}(r)$.

(8) end if

(9) end for

(10) return $\gamma_{r}$.

Algorithm 2: The receiver SH generation algorithm (ES-SH).

where $S_{x}=\{1,2, \ldots, 5\}$ is not rotated. Then the first round of the $\mathrm{PS}_{X} \mathrm{SH}$ sequence with a length 5 is $\left[\begin{array}{lllll}1 & 2 & 3 & 4 & 5\end{array}\right]$. This round is repeated many times, where $\mathrm{SU}_{X}$ continues steering its directional antenna into its sectors as shown in Figure 2(a) to achieve sector rendezvous with its receiver $\mathrm{SU}_{Y}$. On the other hand, the sender $S U_{V}$ in Figure $1(b)$ has $N_{V}=4$ number of sectors, so it selects $P_{V}=5$ as the smallest prime which is larger than $N_{V} . S_{V}$ is rotated to start from 3 and hence $S_{v}=\{3,4,2,1\}$. Then the first round of the $\mathrm{PS}_{V} \mathrm{SH}$ sequence which has adjusted length equal to $P_{v}=5$ is $\left[\begin{array}{llll}3 & 4 & 2 & 1\end{array}\right]$, where $r$ indicates a randomly selected sector from $S_{V}$. The $\mathrm{PS}_{V} \mathrm{SH}$ sequence is constructed as many rounds as shown in Figure 2(b), where $\mathrm{SU}_{V}$ keeps hopping on its sectors to achieve sector rendezvous with the receiver $\mathrm{SU}_{Z}$.

3.1.2. Receiver Sequence in PES-SH. If a SU has nothing to transmit, it serves as a receiver and generates an even-based (ES-SH) sequence as follows. First, randomly select a starting sector index $j \in\left[1, N_{r}\right]$ and circularly rotate the sector set $S=\left\{1, \ldots, N_{r}\right\}$ starting from $j$ as $S=\operatorname{rotate}(S, j-1)$. Second, adjust $N_{r}$ to be the smallest even number $E_{r}$ which is not smaller than $N_{r}$ and is not in the Avoided Even Set (AES). Finally, construct each round of the ES sequence which has a length equal to $E_{r}$ as follows: the $k_{\text {th }}$ sector index of the ESSH round is $S(k)$ if $k \leq N_{r}$; otherwise the $k_{\mathrm{th}}$ sector is selected randomly form $S$.

Consider the receiver $\mathrm{SU}_{Y}$ in Figure 1(a) which has an even number of sectors $N_{Y}=4$ which is not in AES and does not rotate its $S_{Y}$ set. The round of the $\mathrm{ES}_{Y} \mathrm{SH}$ sequence with a length 4 is $\left[\begin{array}{llll}1 & 2 & 3 & 4\end{array}\right]$. This round is repeated many times by $\mathrm{SU}_{Y}$ in order to be paired by a sender as shown in Figure 2(a). On the other hand, the receiver $\mathrm{SU}_{Z}$ in Figure $1(\mathrm{~b})$ has $N_{Z}=3$ sectors; hence it finds $E_{Z}=4$ as the smallest even number larger than $N_{Z}$ and not in AES. $S U_{Z}$ rotates its $S_{Z}$ to start from sector 2 and hence $S_{V}=\{2,3,1\}$. Accordingly, a round of the $\mathrm{ES}_{Z} \mathrm{SH}$ sequence with a length adjusted to $E_{z}=4$ is $\left[\begin{array}{llll}2 & 3 & 1 & r\end{array}\right]$, where $r$ is a randomly selected sector from $S_{z}$. The $\mathrm{ES}_{Z} \mathrm{SH}$ sequence is constructed in rounds as shown in Figure 2(b).

3.2. Scheme Analysis. In this subsection, we study the theoretical performance of the PES-SH, where the MSRL between any two arbitrary SUs performing the PES-SH is derived.

Theorem 1. The MSRL under the PES-SH scheme is $\left(P_{s} E_{r}\right)$, where $P_{s}$ and $E_{r}$ denote the adjusted prime and even numbers of the sectors for the sender and receiver SUs, respectively.

Proof. Suppose that the sector rendezvous pair is $\left(h_{s}, h_{r}\right) \in$ $\left\{1,2, \ldots, N_{s}\right\} \times\left\{1,2, \ldots, N_{r}\right\}$, where $\times$ here indicates the Cartesian product of the two SUs sectors sets. Without loss of generality, we assume that $P_{s}<E_{r}$. Also, we assume that the receiver $\mathrm{SU}_{r}$ starts its $\mathrm{SH}$ sequence with $\delta$ sector slots earlier than the start of the sender $\mathrm{SU}_{s}$. Accordingly, in each PS-SH period of $\mathrm{SU}_{s}$, the $\mathrm{ES}-\mathrm{SH}$ sequence of $\mathrm{SU}_{r}$ is rotate $\left(\mathrm{ES}_{r}, \delta\right)$. In the first round of the $\mathrm{SH}$, when the sender $\mathrm{SU}_{s}$ is on its $h_{s}$ sector, the receiver $\mathrm{SU}_{r}$ is on the $\left(u_{1}=u_{0} \bmod E_{r}+1\right)$ sector, where $1 \leq u_{0} \leq N_{r}$. During the subsequent $E_{r}-1$ rounds, while the sender SU is on its $h_{s}$ sector, the receiver $\mathrm{SU}_{r}$ must be sequentially on $u_{2}=\left(\left(u_{0}+P_{s}\right) \bmod E_{r}+1\right), u_{3}=$ $\left(\left(u_{0}+2 P_{s}\right) \bmod E_{r}+1\right), \ldots, u_{E_{r}}=\left(\left(u_{0}+\left(E_{r}-1\right) P_{s}\right) \bmod E_{r}+1\right)$. As $P_{s}$ and $E_{r}$ are relatively prime, it can be easily proven with the help of the Chinese Reminder Theorem [31] that these $u_{1}, u_{2}, \ldots, u_{E}$ sectors where the receiver resides in the $E_{r}$ rounds are all different. Hence, there must exist a sector among them which is equal to $h_{r}$. According to that and since every round of the PS-SH contains $P_{s}$ sector hops, the SRL required to guarantee a sector rendezvous is upper bound by $P_{s} E_{r}$.

\section{Interleaved Prime- and Even-Based Sequences Sector Hopping (IPES-SH) Scheme}

In the previous section, the PES-SH scheme was designed using the asymmetric approach which requires that each SU have a preassigned role as either a sender or a receiver. In this section, we introduce our interleaved prime- and evenbased sequences (IPES-SH) scheme, which is symmetric (i.e., no preassigned role assumption).

4.1. Scheme Design. In our symmetric IPES-SH scheme, every $\mathrm{SU}$ generates its $\mathrm{SH}$ sequence with the help of a binary 


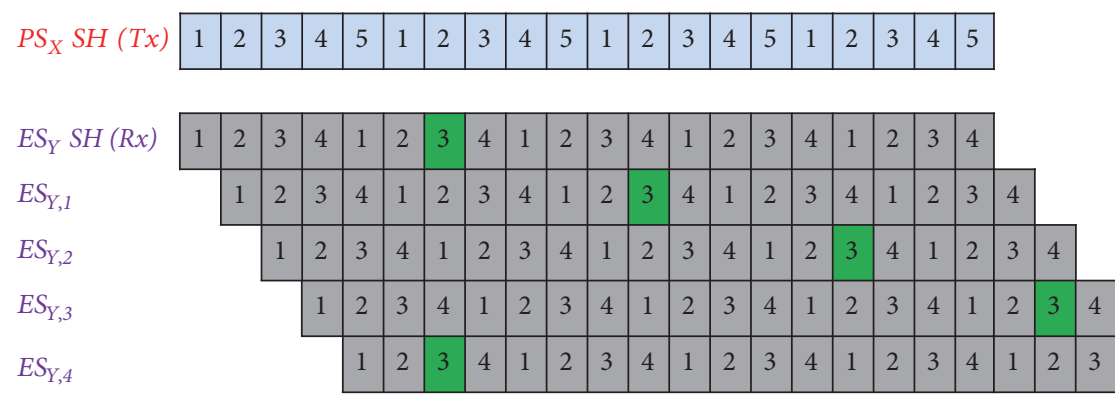

(a) Sector rendezvous between the sender $X$ and the receiver $Y$ on the rendezvous sector pair $(2,3)$ for the cases when $Y$ starts its $\mathrm{SH}$ with $i$ slots later than $X$, where $i \in\left[0, \ldots,\left(P_{X}-1\right)\right]$

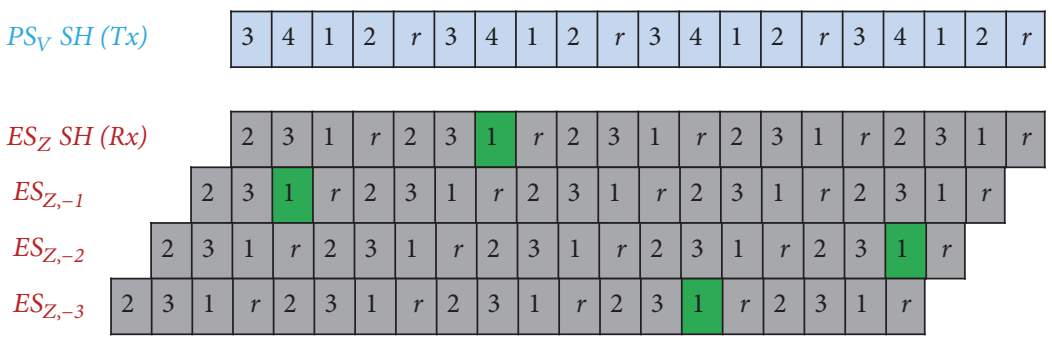

(b) Sector rendezvous between the sender $V$ and the receiver $Z$ on the rendezvous sector pair $(4,1)$ for the cases when $Z$ starts SH with $i$ slots earlier, where $i \in\left[0, \ldots,\left(E_{Z}-1\right)\right]$

FIGURE 2: Successful synchronous and asynchronous sector rendezvous for the two pairs of communicating SUs in Figure 1.

bit sequence such as its globally unique ID. The SH sequence is constructed as an interleaved sequence of several PS and ES SH sequences through a two-step approach. Firstly, each SU transforms its unique ID according to a certain design into another ID sequence so that it is cyclically unique; that is, the resulting ID sequences of any two SUs are cyclic rotationally distinct to each other. Secondly, the SU replaces any bit of 1 (or 0) in its new ID sequence with a PS-based (or ESbased) SH sequence. Accordingly, for any pair of SUs, the cyclic uniqueness property will guarantee the existence of some time instances, where the two SUs are playing different roles. Hence, the sector rendezvous is achieved when one SU is hopping according to its PS sequence while the other $\mathrm{SU}$ is hopping based on its ES sequence and vice versa.

Several methods have been proposed in the literature to transform the unique ID into another cyclically unique binary sequence. In [32], a method was proposed to expand the $m$-bit ID into another $3 m$-bit extended ID that is cyclically unique. The unique $m$-bit ID in that method is expanded by appending $m$-bit consecutive 0 's into the beginning of the ID and other $m$-bit consecutive l's at the end of the ID. Another method for generating cyclically unique IDs that are used for the construction of symmetric role $\mathrm{SH}$ sequences has been proposed in [30]. The method appends the original $m$-bit ID with other $(m+1)$-bits of consecutive 0 's and 1's, which results in $(2 m+1)$-bits expanded ID sequence. However, the extended ID sequences generated by these methods are relatively long, especially for the former method. This results in long rendezvous delay when they are used for constructing symmetric role sequences. Therefore, we propose an alternative method for constructing cyclic rotationally distinct sequences, which provides shorter extended ID lengths than the methods in $[30,32]$. So, it provides shorter sector rendezvous delay when it is used for constructing symmetric role sequences in our IPES-SH scheme.

4.1.1. Constructing the Cyclic Unique ID Sequences. In our method, each $m$-bit ID is extended by appending only $m$-bits of 1's and 0's in the beginning and in the end of the ID.

Lemma 2. Given any two $m$-bit ID sequences $\alpha=\left\{\alpha_{1}, \ldots, \alpha_{m}\right\}$ and $\beta=\left\{\beta_{1}, \ldots, \beta_{m}\right\}$, let $\mathbf{a}$ and $\mathbf{b}$ be two $2 m$-bit expanded ID sequences generated from $\alpha$ and $\beta$ as follows:

$\mathbf{a} \stackrel{\text { def }}{=} \mathbf{1}(\lceil\mathrm{m} / 2\rceil)\|\alpha\| \mathbf{0}(\lfloor\mathrm{m} / 2\rfloor)$ and $\mathbf{b} \stackrel{\text { def }}{=} \mathbf{1}(\lceil\mathrm{m} /$ $2\rceil)\|\beta\| \mathbf{0}(\lfloor m / 2\rfloor)$, where $\mathbf{1}(\cdot)$ is a bit sequence composed of only l's and $\mathbf{0}(\cdot)$ is a bit sequence composed of only 0 's. Then $\mathbf{a}$ and $\mathbf{b}$ are cyclic rotationally distinct from each other, which means that

$$
\text { If } \mathbf{a} \neq \mathbf{b} \Longrightarrow \mathbf{a} \neq \operatorname{rotate}(\mathbf{b}, k), \quad \forall k \in(0,2 m-1] .
$$

Proof. To prove the above lemma, we consider all the possible cases that may happen when sequence $b$ is rotated with $(k \in$ $(0,2 m-1])$. We show in each case that bits in sequence $\mathbf{a}$ and other bits in sequence $b^{\prime}=$ rotate $(\mathbf{b}, k)$ have different values, even though these bits are in the same positions. Considering the five cases in Figure 3, it is sufficient to prove that $\mathbf{a}$ and $\mathbf{b}^{\prime}=$ rotate $(\mathbf{b}, k)$ are cyclic rotationally distinct to each other.

Case $1(k \in(0,[m / 2\rceil])$. As indicated by the red arrow in Figure 3 , it holds that $a_{2 m}=0$ and $b_{2 m}^{\prime}=1$. 


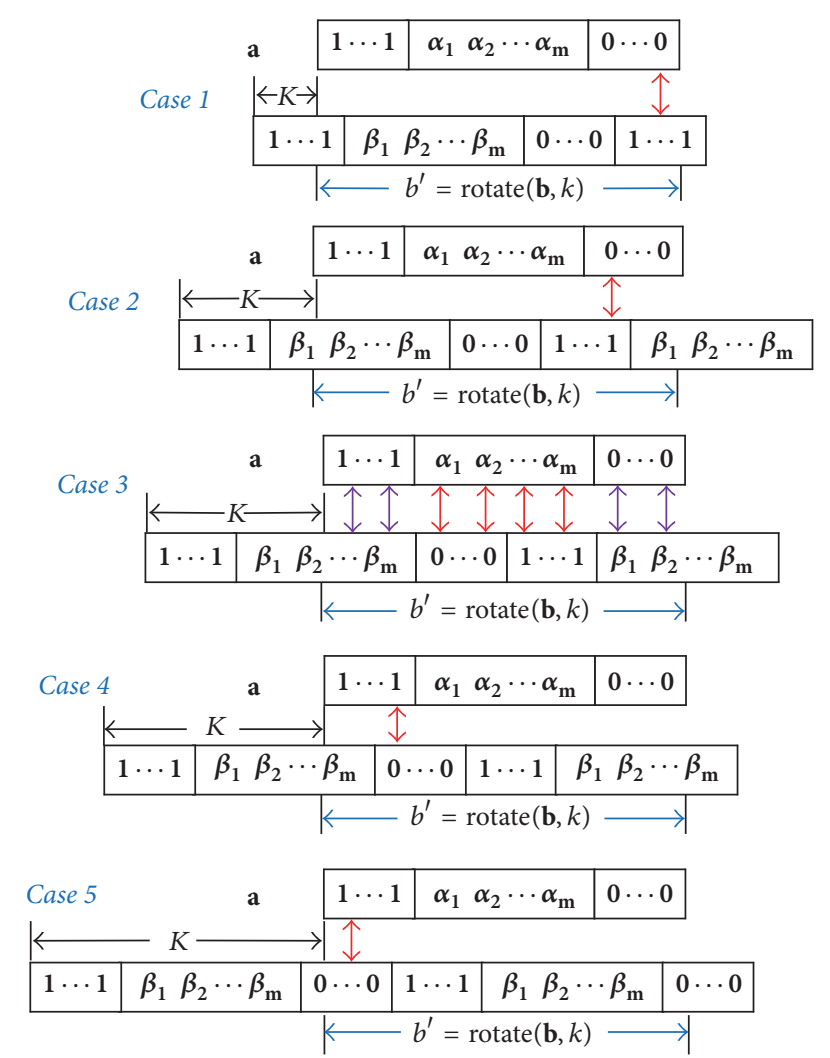

FIgURE 3: Illustration of the five cases in the proof of Lemma 2.

Case $2(k \in(\lceil m / 2\rceil, m))$. As indicated by the red arrow in Figure 3, it holds that $a_{([m / 2]+m+1)}=0$ and $b_{([m / 2]+m+1)}^{\prime}=1$.

Case $3(k=m)$. As indicated by the $m$ red arrows in Figure 3 , the $m$ bits of sequence a starting from $a_{([m / 2]+1)}$ are $\left\{\alpha_{1}, \ldots, \alpha_{m-1}\right\}$, while the $m$ bits of $b^{\prime}$ in the same positions are $\mathbf{0}(\lfloor m / 2\rfloor) \| \mathbf{1}([m / 2\rceil)$. On the other hand, as indicated by the $m$ purple arrows in Figure 3, the first $\lceil m / 2\rceil$ bits of sequence a are l's and the last $\lfloor m / 2\rfloor$ are 0 's, whereas the first $\lceil m / 2\rceil$ bits of $b^{\prime}$ are $\left\{\beta_{([m / 2\rceil)}, \ldots, \beta_{m}\right\}$ and the last $\lfloor m / 2\rfloor$ bits are $\left\{\beta_{1}, \ldots, \beta_{(\lfloor m / 2\rfloor)}\right\}$. And because $\alpha \neq \beta$, it holds that there must be at least a single bit that is different in $a$ and $b^{\prime}$.

Case $4(k \in(m, m+\lfloor m / 2\rfloor])$. As indicated by the red arrow in Figure 3, it holds that $a_{([m / 2])}=1$ and $b_{([m / 2])}^{\prime}=0$.

Case $5(k \in(m+\lfloor m / 2\rfloor, 2 m-1])$. As indicated by the red arrow in Figure 3, it holds that $a_{1}=1$ and $b_{1}^{\prime}=0$.

According to these possible cases, we conclude that $\mathbf{a} \neq$ rotate $(\mathbf{b}, k), \forall k \in(0,2 m-1]$. In Figure 4 , we give an example for two ID sequences of original lengths $m=4$ which are expanded to $m=8$ bits. The figure illustrates the cyclic uniqueness property between the IDs for all the rotation cases (i.e., $\forall k \in[0,7])$.

4.1.2. Generating the Interleaved Sector Hopping Sequence. We now explain how to generate the symmetric IPES-SH sequence for any $\mathrm{SU}$, say $\mathrm{SU}_{i}$. Let the number of sectors for

\begin{tabular}{|l|l|l|l|l|l|l|l|}
\hline 1 & 1 & $\alpha_{1}$ & $\alpha_{2}$ & $\alpha_{3}$ & $\alpha_{4}$ & 0 & 0 \\
\hline
\end{tabular}

\begin{tabular}{|c|c|c|c|c|c|c|c|c|c|}
\hline b & 1 & 1 & $\beta_{1}$ & $\beta_{2}$ & $\beta_{3}$ & $\beta_{4}$ & 0 & 0 & \\
\hline$b^{\prime}=\operatorname{rot}(\mathbf{b}, 1)$ & 1 & $\beta_{1}$ & $\beta_{2}$ & $\beta_{3}$ & $\beta_{4}$ & 0 & 0 & 1 & \multirow{2}{*}{ - Case 1} \\
\hline$b^{\prime}=\operatorname{rot}(\mathbf{b}, 2)$ & $\beta_{1}$ & $\beta_{2}$ & $\beta_{3}$ & $\beta_{4}$ & 0 & 0 & 1 & 1 & \\
\hline$b^{\prime}=\operatorname{rot}(\mathbf{b}, 3)$ & $\beta_{2}$ & $\beta_{3}$ & $\beta_{4}$ & 0 & 0 & 1 & 1 & $\beta_{1}$ & Case 2 \\
\hline$b^{\prime}=\operatorname{rot}(\mathbf{b}, 4)$ & $\beta_{3}$ & $\beta_{4}$ & 0 & 0 & 1 & 1 & $\beta_{1}$ & $\beta_{2}$ & Case 3 \\
\hline$b^{\prime}=\operatorname{rot}(\mathbf{b}, 5)$ & $\beta_{4}$ & 0 & 0 & 1 & 1 & $\beta_{1}$ & $\beta_{2}$ & $\beta_{3}$ & \multirow{2}{*}{ - Case 4} \\
\hline$b^{\prime}=\operatorname{rot}(\mathbf{b}, 6)$ & 0 & 0 & 1 & 1 & $\beta_{1}$ & $\beta_{2}$ & $\beta_{3}$ & $\beta_{4}$ & \\
\hline$b^{\prime}=\operatorname{rot}(\mathbf{b}, 7)$ & 0 & 1 & 1 & $P_{1}$ & $\beta_{2}$ & $\beta_{3}$ & $\beta_{4}$ & 0 & Case 5 \\
\hline
\end{tabular}

FIGURE 4: An illustrative example for the correctness of our cyclic rotationally distinct sequences construction method when $m=4$.

$\mathrm{SU}_{i}$ be $N_{i}$ and hence the sector set $S_{i}=\left\{1, \ldots, N_{i}\right\}$. Suppose that $\mathrm{SU}_{i}$ has $m$-bits globally unique ID sequence, $\alpha$, which is extended by our above method to $2 m$-bits sequence $\mathbf{a}=$ $\mathbf{1}([m / 2\rceil)\|\alpha\| \mathbf{0}(\lfloor m / 2\rfloor)$.

(i) Select randomly a starting index $k \in\left[1, N_{i}\right]$ and generate a rotated set $S_{i}^{P}$ by rotating $S_{i}$ circularly starting from $k$ as $S_{i}^{P}=\operatorname{rotate}\left(S_{i}, k-1\right)$. Also, generate another sector indices set as a reverse of $S_{\mathrm{i}}^{P}$ which is $S_{i}^{E}=\operatorname{Reverse}\left(S_{i}^{P}\right)$.

(ii) Find $\left(P_{i}: P_{i}>3\right)$ as the smallest prime number that is not smaller than $N_{i}$. Also find $\left(E_{i}: E_{i} \notin \mathrm{AES}\right)$ as the smallest even number that is not smaller than $N_{i}$.

(iii) Define an empty matrix $M_{i}$ that has $2 m$ columns and $P_{i} \times E_{i}$ rows.

(iv) Fill the matrix by mapping each bit in a to a certain column as described in Algorithm 3.

(v) Generate the $\mathrm{SH}$ sequence by concatenating the matrix rows (row by row). $\mathrm{SU}_{i}$ keeps hopping according to this generated $\mathrm{SH}$ sequence and repeats it in order to rendezvous with its pair partner SU.

Figure 5 illustrates the IPES-SH sequence construction matrices for $\mathrm{SU}_{V}$ and $\mathrm{SU}_{Z}$ in our previous example in Section 3.1 (Figure 1(b)). In Figure 5, the ID sequence of $\mathrm{SU}_{V}$ that has $N_{v}=4$ sectors is $\alpha=\{1,0\}$ which is expanded to $\mathbf{a}=$ $\{1,1,0,0\}$. On the other side, the ID sequence of $\mathrm{SU}_{V}$ that has $N_{z}=3$ sectors is $\beta=\{0,1\}$ which is expanded also to $\mathbf{b}=\{1,0,1,0\}$. The constructed matrix for $\mathrm{SU}_{V}$ has $P_{v} \times$ $E_{v}=5 \times 4=20$ rows and $2 m=4$ columns. Similarly, $\mathrm{SU}_{Z}$ constructs its matrix with $P_{z} \times E_{z}=5 \times 4=20$ rows and $2 m=4$ columns. Each SU will assign its matrix columns with either PS sequence or ES sequence based on the corresponding bits of its extended ID sequence. For example, since the first bit of $\mathrm{SU}_{V}$ extended ID is one, $\mathrm{SU}_{V}$ firstly 
(1) for $c=1: 2 m$ do

(2) if $\left(a_{c}==1\right)$ then

(3) Invoke Algorithm 1 with $S_{i}^{P}$ and $N_{i}$ to construct a round $\omega$ of an PS sequence.

(4) generate $\omega^{\prime}$ by repeating $\omega$ for $E_{i}$ times.

(5) Map the $c_{\text {th }}$ column of the matrix with $\omega^{\prime}$.

(6) $S_{i}^{P}=\operatorname{Rotate}\left(S_{i}^{P}, 1\right)$.

(7) else

(8) Invoke Algorithm 2 with $S_{i}^{E}$ and $N_{i}$ to construct a round $\gamma$ of an ES sequence.

(9) generate $\gamma^{\prime}$ by repeating $\gamma$ for $P_{i}$ times.

(10) Map the $c_{\text {th }}$ column of the matrix with $\gamma^{\prime}$.

(11) $S_{i}^{E}=\operatorname{Rotate}\left(S_{i}^{E}, 1\right)$.

(12) end if

(13) end for

Algorithm 3

generates its first sector sets as $S_{v}^{P}=\{3,4,1,2\}$ and then invokes Algorithm 1 with $S_{v}^{P}$ and $N_{v}=4$ to construct a round of a PS sequence $\omega=\left[\begin{array}{lllll}3 & 4 & 1 & 2 & r\end{array}\right]$. This round $\omega$ is repeated for $E_{v}=4$ times and assigned to the first column of $\mathrm{SU}_{V}$ matrix. Similarly, since the second bit of $\mathrm{SU}_{V}$ extended ID is one, the second column of $\mathrm{SU}_{V}$ matrix is assigned with a repeated sequence of the PS sequence [ $\left[\begin{array}{lllll}4 & 1 & 2 & 3 & r\end{array}\right]$, which is constructed after rotating the sector set $S_{v}^{P}$ by one. On the other hand, $\mathrm{SU}_{V}$ assigns the third and fourth columns of its matrix with ES sequences because the corresponding $3_{\text {rd }}$ and $4_{\text {th }}$ bits of its extended ID sequence are zeros. Specifically, $\mathrm{SU}_{V}$ invokes Algorithm 2 with $S_{v}^{E}=\operatorname{Reverse}\left(S_{v}^{P}\right)$ and $N_{v}=4$ to construct a round of an ES sequence $\gamma=\left[\begin{array}{lll}2 & 1 & 4\end{array}\right]$. This round $\gamma$ of the ES sequence is then repeated for $P_{v}=5$ times and assigned to the third column of $\mathrm{SU}_{V}$ matrix. Similarly, the fourth column of $\mathrm{SU}_{V}$ matrix is filled with the ES sequence $\left[\begin{array}{llll}1 & 4 & 3 & 2\end{array}\right]$ after repeating it for $P_{v}=5$ times. Note that $\mathrm{SU}_{Z}$ will follow the same procedures as $\mathrm{SU}_{V}$ to construct its matrix with taking into account the different expanded ID sequence $b_{z}=\{1,0,1,0\}$ and $N_{z}$, as well as the initial sector sets $S_{z}^{P}$ and $S_{z}^{E}$

Figure 6 shows partial sector slots of the IPES-SH sequences for the two SUs $\left(\mathrm{SU}_{V}\right.$ and $\left.\mathrm{SU}_{Z}\right)$. As depicted from the figure, the IPES-SH sequence of each SU is generated by concatenating the rows of the SU constructed matrix in Figure 5 row by row. The sequences are generated as interleaved slots of PS (light blue) and ES (gray) slots according to the respective columns types in the matrices. The figure also illustrates the rendezvous sector occurrence between the SUs (indicated by the green circle) on the sector rendezvous pair $(4,1)$.

4.2. Scheme Analysis. In this subsection, the theoretical performance of IPES-SH scheme is studied where the MSRL is derived.

Theorem 3. Under the IPES-SH scheme, the MSRL between a pair of $S U s\left(S U_{i}\right.$ and $\left.S U_{j}\right)$ is $2 m \times\left(\max \left\{\left(P_{i} E_{j}\right),\left(E_{i} P_{j}\right)\right\}\right)$.

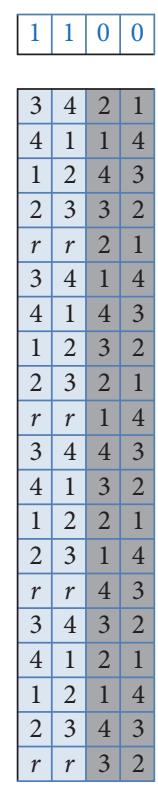

(a) IPES matrix $M_{V}$ for $\mathrm{SU}_{V}$

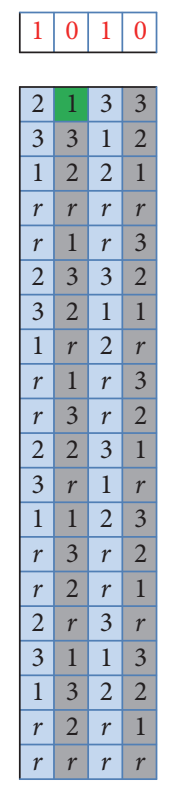

(b) IPES matrix $M_{Z}$ for $\mathrm{SU}_{Z}$
FIgURE 5: The construction of the IPES matrices $M_{V}$ and $M_{Z}$ for $\mathrm{SU}_{V}$ and $\mathrm{SU}_{Z}$, respectively. $N_{V}=4$ and $N_{Z}=3 ; \alpha_{V}=\{1,0\}$ and $\beta_{Z}=\{0,1\}$. So the expanded ID sequences are $\mathbf{a}_{V}=\{1,1,0,0\}$ and $\mathbf{b}_{Z}=\{1,0,1,0\}$.

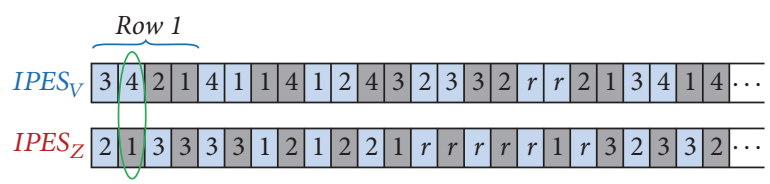

FIGURE 6: IPES-SH sector rendezvous between $\mathrm{SU}_{V}$ and $\mathrm{SU}_{Z}$ for the synchronous case.

Proof. To prove that IPES-SH has MSRL $=2 m\left(\max \left\{\left(P_{i} E_{j}\right)\right.\right.$, $\left.\left.\left(E_{i} P_{j}\right)\right\}\right)$, it is sufficient to prove that any arbitrary pair of IPES$\mathrm{SH}$ sequences, for example, $\mathrm{IPES}_{i}$ and $\mathrm{IPES}_{j}$, can achieve a successful sector rendezvous within $2 m\left(\max \left\{\left(P_{i} E_{j}\right),\left(E_{i} P_{j}\right)\right\}\right)$ sector slots.

Let IPES ${ }_{i}$ and IPES $_{j}$ be the corresponding IPES-SH sequences of IPES matrices $M_{i}$ and $M_{j}$ (i.e., IPES Ind IPES $_{j}$ are generated by concatenating the rows of matrices $M_{i}$ and $M_{j}$, resp.). For example, Figures 5(a) and 5(b) show the IPES matrices $M_{V}$ and $M_{Z}$, respectively. Without loss of generality, assume that $\mathrm{SU}_{j}$ starts its $\mathrm{SH}$ sequence $\delta$ sector slots earlier than the start of $\mathrm{SU}_{i}$. In each $\mathrm{SH}$ period of $\mathrm{SU}_{i}$, the $\mathrm{SH}$ sequence of $\mathrm{SU}_{j}$ is rotate $\left(\mathrm{IPES}_{j}, \delta\right)$. Clearly, the rendezvous between this pair of SUs is the same as the rendezvous of $M_{i}$ and $M_{(j, \delta)}$. Suppose that the sector rendezvous pair is $\left(h_{i}, h_{j}\right) \in\left\{1,2, \ldots, N_{i}\right\} \times\left\{1,2, \ldots, N_{j}\right\}$. The rendezvous of the matrices is a pair of entries in the matrices whose value in $M_{i}$ is $h_{i}$ and its values in $M_{(j, \delta)}$ is $h_{j}$. Without loss of generality, suppose that the sector slot shift $\delta=2 m \times \delta_{R}+\delta_{C}$, where $\delta_{R} \in\left[0,\left(P_{j} \times E_{j}\right)-1\right]$, is the shift of row and $\delta_{C} \in[0,(2 m)-1]$ is the shift of column. Below, we consider the two different cases for rendezvous of IPES $i$ and IPES $_{j}$. 


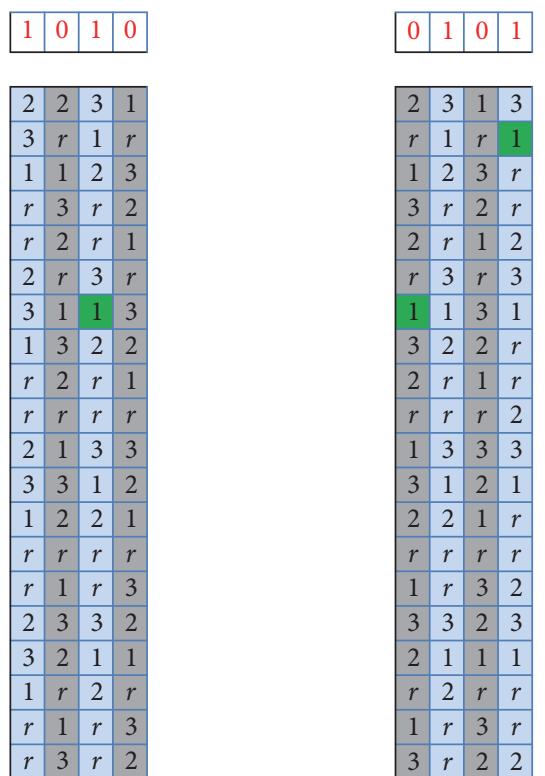

(a) $M_{(Z, 40)}, \delta_{C}=0$, and $\delta_{R}=10 \quad$ (b) $M_{(Z, 41)}, \delta_{C}=1$, and $\delta_{R}=10$

FIGURE 7: The IPES matrix $M_{Z}$ for $\mathrm{SU}_{Z}$ when it is circularly rotated with (a) $\delta=2 m \times 10+0=40$ sector slots and (b) $\delta=2 m \times 2+1=41$ slots.

Case $1\left(\delta_{C}=0\right)$. In this case, it is implied that there is no column shift. Recall that the expanded ID sequences of $\mathrm{SU}_{i}$ and $\mathrm{SU}_{j}$ are distinct. Since the columns types of the matrices are assigned based on these distinct expanded IDs, it is guaranteed that there must exist at least a single common column $f$ in both matrices which is a PS-column in one matrix and an ES-column in the other matrix, and vice versa. In other words, the $f_{\text {th }}$ column of $M_{i}$ contains a PS-SH sequence (reps., ES-SH), while the same position $f_{\text {th }}$ column in $M_{(j, \delta)}$ contains an ES-SH (resp., PS-SH). By Theorem 1, we know that when $\mathrm{SU}_{i}$ and $\mathrm{SU}_{j}$ repeat the PS$\mathrm{SH}$ (resp., ES-SH) and the ES-SH (resp., PS-SH) sequences, respectively, they rendezvous within $P_{i} \times E_{j}$ (resp., $E_{i} \times P_{j}$ ). Meanwhile, since the elements of a specific column of each matrix appear in the corresponding IPES-SH sequence every $2 m$ slots, for any value of the row shift $\delta_{R}, M_{i}$ and $M_{(j, \delta)}$ are guaranteed to rendezvous on their $f_{\text {th }}$ columns no later than $2 m \times \max \left\{\left(P_{i} E_{j}\right),\left(E_{i} P_{j}\right)\right\}$. Specifically, the rendezvous happens when an entry of the $f_{\text {th }}$ column in $M_{i}$ is $h_{i}$, while the same position entry of the $f_{\text {th }}$ column in $M_{(j, \delta)}$ is $h_{j}$. For example, Figure 5 shows the rendezvous of $M_{V}$ and $M_{Z}$ for the synchronous case where the slots shift $\delta=2 m \times 0+0=0$. The sector rendezvous occurs between the second columns of $M_{V}$ and $M_{Z}$ which is indicated by the green entry in $M_{Z}$. However, Figure $7(\mathrm{a})$ shows the IPES matrix of $\mathrm{SU}_{Z}$ when it is shifted with row shift $\delta_{R}=10$. Hence, this means that $\delta=2 m \times 10+0=40$. The rendezvous between $M_{V}$ in Figure 5 and $M_{(Z, 40)}$ in Figure $7(\mathrm{a})$ is achieved at their third columns.

Case $2\left(\delta_{C} \neq 0\right)$. In this case, it holds that there is a column shift $\delta_{C} \in[1,(2 m-1)]$. Let $\mathbf{a}$ and $\mathbf{b}$ be the expanded ID sequences of $\mathrm{SU}_{i}$ and $\mathrm{SU}_{j}$, respectively. By Lemma 2, we know that $\mathbf{a}$ and $\mathbf{b}^{\prime}=\operatorname{rotate}(\mathbf{b}, k)$, where $k \in(2 m-1)$ are cyclic rotationally distinct to each other. Thus, for a column shift $\delta_{C} \in[1,(2 m-1)]$ of matrix $M_{j}$, there must exist a column $f$ in matrix $M_{i}$ which has different type than the same position $f_{\text {th }}$ column in the shifted matrix $M_{\left(j, \delta_{C}\right)}$. Accordingly, the rendezvous is guaranteed to occur at the $f_{\text {th }}$ columns in the matrices, despite any value of the row shift $\delta_{R}$. For example, Figure 7 (b) shows the shifted IPES matrix of $\mathrm{SU}_{Z}$ when the column shift $\delta_{C}=1$ and the row shift $\delta_{R}=10$, which results in $\delta=2 m \times 10+1=41$ slots. The first rendezvous between $M_{V}$ in Figure 5 and $M_{(Z, 41)}$ in Figure $7(\mathrm{~b})$ is achieved at the forth columns.

Summarizing the discussion above, we show that, in IPES-SH, any two SUs are guaranteed to rendezvous on their sector rendezvous pair within $\mathrm{SH}$ period $2 m \times$ $\max \left\{\left(P_{i} E_{j}\right),\left(E_{i} P_{j}\right)\right\}$.

\section{Combined Sector and Channel Hopping Schemes}

While we explained in the previous sections the procedures of our SH schemes by which the SUs keep steering their antennas in order to achieve sector rendezvous with their communicating partners, the scheme for channel hopping $(\mathrm{CH})$ exposed by the SUs in their sectors for achieving a channel rendezvous between them was not explained. In this section, the design of the exposed $\mathrm{CH}$ scheme and the procedure for the combined sector and channel hopping (SCH) schemes are presented. The key for the design of the exposed $\mathrm{CH}$ scheme is to construct two different types of $\mathrm{CH}$ sequences which will be combined within the two different types of PES-SH sequences. These $\mathrm{CH}$ sequences must guarantee a successful channel rendezvous between the pair of SUs as long as they achieve a successful sector rendezvous (i.e., directing their antenna beams towards each other). Furthermore, the $\mathrm{CH}$ sequences should be designed in such a way that the pair of communicating SUs stay for a similar period (i.e., number of time slots) in each sector of their $\mathrm{SH}$ sequences. By doing this, the pair of communicating SUs are guaranteed to achieve successful sector and channel rendezvous within a bounded and short time, despite any asynchronous time offsets between their local clocks.

5.1. Asymmetric Grid Quorum-Based Channel Hopping. In this subsection, we explain the design of our Asymmetric Grid Quorum-based Channel Hopping (AGQ-CH) scheme that is executed by the SUs in each sector of their $\mathrm{SH}$ sequences. The AGQ-CH scheme utilizes the grid quorum system (GQS) in asymmetric-role design, where two types of $\mathrm{CH}$ sequences are constructed. These types of $\mathrm{CH}$ sequences will be combined within the two different types of our $\mathrm{SH}$ sequences. Some preliminary definitions related to grid quorum systems are presented first to facilitate the understanding of the $\mathrm{CH}$ scheme.

Definition 4. For a set $\mathbb{Z}_{n}=\{1,2, \ldots, n\}$, a quorum system $Q$ under $\mathbb{Z}_{n}$ is a group of nonempty subsets of $\mathbb{Z}_{n}$, each called a quorum, such that $\forall X$ and $Y \in Q, X \cap Y \neq \emptyset$. Here, $\mathbb{Z}_{n}$ is the set of integers less than or equal to $n$. 
Definition 5. A GQS arranges the elements of $\mathbb{Z}_{n}$ as a $\sqrt{n} \times \sqrt{n}$ square grid array, where $n$ must be a square of a positive integer. Hence, a quorum is formed as a union of the elements of one column and one row of the grid. There are $n$ grid quorums in a GQS that is constructed under $\mathbb{Z}_{n}$ and each quorum has a size of $(2 \times \sqrt{n}-1)$.

For example, a GQS $Q$ under $\mathbb{Z}_{n}=\{1,2,3,4\}$ is $Q=$ $\left\{Q_{1}, \ldots, Q_{4}\right\}=\{\{1,2,3\},\{1,2,4\},\{1,3,4\},\{2,3,4\}\}$.

Definition 6. For a given integer $i$ and a grid quorum $\mathscr{G}$ in a GQS $Q$ under $\mathbb{Z}_{n}$, we define rotate $(\mathscr{G}, i)=\{(x+i) \bmod n, x \in$ $\mathscr{G}$ \} to denote a cyclic rotation of quorum $\mathscr{G}$ by $i$.

Definition 7. A GQS Q under $\mathbb{Z}_{n}$ satisfies the rotation closure property because, $\forall \mathscr{G}_{i 1}, \mathscr{G}_{i 2} \in Q$ and $\forall j_{1}, j_{2} \in \mathbb{Z}_{n}$, $\operatorname{rotate}\left(\mathscr{G}_{i 1}, j_{1}\right) \cap \operatorname{rotate}\left(\mathscr{G}_{i 2}, j_{2}\right) \neq \emptyset$.

GQSs have been utilized to construct $\mathrm{CH}$ sequences that achieve asynchronous communications because they satisfy the intersection and the rotation closure properties [33,34]. In the conventional GQS, each grid quorum is formed as a union of the elements of one column and one row of the grid array. However, different from the conventional GQS, our asymmetric GQS (AGQS) uses semigrid quorums. One semiquorum, called the Grid Column-based Quorum (GCQ), is formed from the elements of a single column of the grid. Meanwhile, the other semiquorum, called Grid Row-based Quorum (GRQ), is formed from the elements of a single row in the grid. The nonempty intersection and rotation closure properties in the AGQS are inherited from the conventional GQS. Specifically, for any arbitrary pair of GCQ and GRQ, there must exist one common element between them despite any rotation of the grid.

The GCQs and GRQs in the AGQS are used to construct two asymmetric $\mathrm{CH}$ sequences that can be combined within the different types of sectors in our SH schemes. The constructed $\mathrm{CH}$ sequences are guaranteed to achieve a successful channel rendezvous between a pair of communicating SUs as long as there exists at least a single common channel between the available channel sets of the SUs. In our AGQ-CH scheme, to construct $\mathrm{CH}$ sequences for assigning $n$ channels, $n \times n$ grid array is built. In the GCQ-based CH sequence, the $n$ rows of the grid are used for assigning the $n$ channels into the $\mathrm{CH}$ sequence, where the elements of each row are used to map a single channel. On the other side, the $n$ columns of the grid are used for the assignment of the $n$ channels into the GRQbased $\mathrm{CH}$ sequence. For example, Let $n=4$ be the number of channels for a sender $\mathrm{SU}_{x}$ and a receiver $\mathrm{SU}_{y}$. Suppose that $\mathrm{SU}_{x}$ has the channel set $\mathscr{C}_{x}=\left\{\mathrm{Ch}_{1}, \mathrm{Ch}_{2}, \mathrm{Ch}_{3}, \mathrm{Ch}_{4}\right\}$, while the channel set of $\mathrm{SU}_{y}$ is $\mathscr{C}_{y}=\left\{\mathrm{Ch}_{3}, \mathrm{Ch}_{5}, \mathrm{Ch}_{6}, \mathrm{Ch}_{8}\right\}$. Accordingly, a grid array of size $4 \times 4$ is formed by both SUs as shown in Figure 8. Without loss of generality, assume that the sender uses the 4 GCQs to map its channels into the time slots of its $\mathrm{CH}$ sequence as depicted in Figure 8(a), while the receiver $\mathrm{SU}_{y}$ maps its channels based on the $4 \mathrm{GRQs}$ as depicted in Figure 8(b). The CH sequences for both SUs are shown in Figure 8(c), where they map each channel of their channel sets into the time slots of their $\mathrm{CH}$ sequence according to the selected (GCQ or GRQ) semiquorum. As shown in Figure 8(c), the channel rendezvous is achieved on the common channel $\mathrm{Ch}_{3}$ between the SUs despite the time offsets between their local clocks.

5.2. Channel Ranking. As explained in the beginning of the section, the sector frame length which represents the number of time slots by which every SU spends on each sector for executing $\mathrm{CH}$ should be the same in all SUs. This is in order to guarantee channel rendezvous between any pair of neighbouring SUs side by side with the sector rendezvous despite any time offsets between their local clocks. To achieve this, we let the SUs construct their combined $\mathrm{CH}$ sequences based on the best-quality ( $n \subseteq L$ ) channels. The channel's quality is generally represented by the quantity of the measured PU noise on the channel within the corresponding sector. However, for cases where some of the channels have the same measured PU noise, channels indices are used to differentiate between their ranking levels. Specifically, the bigger the channel index, the higher the quality. We highlight here that the similarity of the measured PU noises for some channels is a practical issue in DIR-CRNs which happen when the channels are idle from any PU activity within the sector area.

The channel ranking is done by each SU before executing the rendezvous process based only on its own view of the available channels in its sectors. Each $\mathrm{SU}_{i}$ ranks the $L$ network channels in each sector $S_{k}$ of its sectors set $S_{i}=$ $\left\{S_{1}, S_{2}, \ldots, S_{N_{i}}\right\}$ based on their qualities within the sector $k$. Thus, a ranking table of size $L \times N_{i}$ is maintained by each $\mathrm{SU}_{i}$ which contains the ranked channels in all the sectors. This table is called Sectors Ranked Channels Table (SRCT), where each column $k$ of it indicates a sector of the $N_{i}$ sectors. These columns contain the $L$ network channels ranked decreasingly from the best to the worst according to their qualities within the corresponding sectors. We note here that since each SU in the DIR-CRN performs the same ranking mechanism, this will increase the probability of having a similar ranked channel list among any pair of neighbouring SUs on their rendezvous sectors.

5.3. Combined SCH Sequence Generation. We now explain the generation procedures for the combined PES-SCH and IPES-SCH sequences.

5.3.1. Combined PES-SCH Scheme. Since our PES-SH scheme is designed based on two different types of coprimality-based sequences (i.e., either PS or ES), we combine each sector of the $\mathrm{SH}$ sequences according to its type with a corresponding type from the two AGQ-CH sequences. Specifically, each sector of the sender PS-SH sequence is combined with a GCQ-CH sequence, while each sector of the receiver ES-SH sequence is combined with a GRQ-CH sequence. Algorithms 4 and 5 describe the SCH generation procedures for the sender and receiver SUs, respectively. As illustrated by the algorithms, each SU will keep hopping on its best $n$ quality channels in each sector of its SH sequence according to the corresponding $\mathrm{CH}$ sequence. This will guarantee that when a successful sector rendezvous occurs between a sector 


\begin{tabular}{|c|c|c|c|}
\multicolumn{1}{c}{$\mathrm{Ch}_{1}$} & $\mathrm{Ch}_{2}$ & $\mathrm{Ch}_{3}$ & $\mathrm{Ch}_{4}$ \\
\hline 1 & 2 & 3 & 4 \\
\hline 5 & 6 & 7 & 8 \\
\hline 9 & 10 & 11 & 12 \\
\hline 13 & 14 & 15 & 16 \\
\hline
\end{tabular}

(a) GCQs for mapping $\mathscr{C}_{x}$

\begin{tabular}{l|c|c|c|c|}
\cline { 2 - 5 } $\mathrm{Ch}_{3}$ & 1 & 2 & 3 & 4 \\
\cline { 2 - 5 } $\mathrm{Ch}_{5}$ & 5 & 6 & 7 & 8 \\
\cline { 2 - 5 } $\mathrm{Ch}_{6}$ & 9 & 10 & 11 & 12 \\
\cline { 2 - 5 } $\mathrm{Ch}_{8}$ & 13 & 14 & 15 & 16 \\
\cline { 2 - 5 } & &
\end{tabular}

(b) GRQs for mapping $\mathscr{C}_{y}$

$$
\begin{aligned}
& \begin{array}{l|l|l|l|l|l|l|l|l|l|l|l|l|l|l|l|l|}
\mathrm{GCQ}_{X} \mathrm{CH} & 1 & 2 & 3 & 4 & 1 & 2 & 3 & 4 & 1 & 2 & 3 & 4 & 1 & 2 & 3 & 4 \\
\hline
\end{array} \\
& \begin{array}{|c|l|l|l|l|l|l|l|l|l|l|l|l|l|l|l|l|}
\mathrm{GRQ}_{Y} \mathrm{CH} & 3 & 3 & 3 & 3 & 5 & 5 & 5 & 5 & 6 & 6 & 6 & 6 & 8 & 8 & 8 & 8 \\
\hline
\end{array}
\end{aligned}
$$

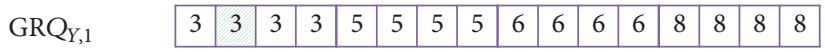

$$
\begin{aligned}
& \begin{array}{|l|l|l|l|l|l|l|l|l|l|l|l|l|l|l|l|l|}
\hline \text { GRQ }_{Y, 5} & 3 & 3 & 3 & 3 & 5 & 5 & 5 & 5 & 6 & 6 & 6 & 6 & 8 & 8 & 8 & 8 \\
\hline
\end{array}
\end{aligned}
$$

(c) Channel rendezvous between $\mathrm{SUs}$ on the common channel $\mathrm{Ch}_{3}$ when $\mathrm{SU}_{y}$ start its $\mathrm{CH}$ with 0,1 , and 5 slots later than $\mathrm{SU}_{x}$

FIGURE 8: An example of AGQ-CH sequences constructions and rendezvous for a pair of communicating SUs.

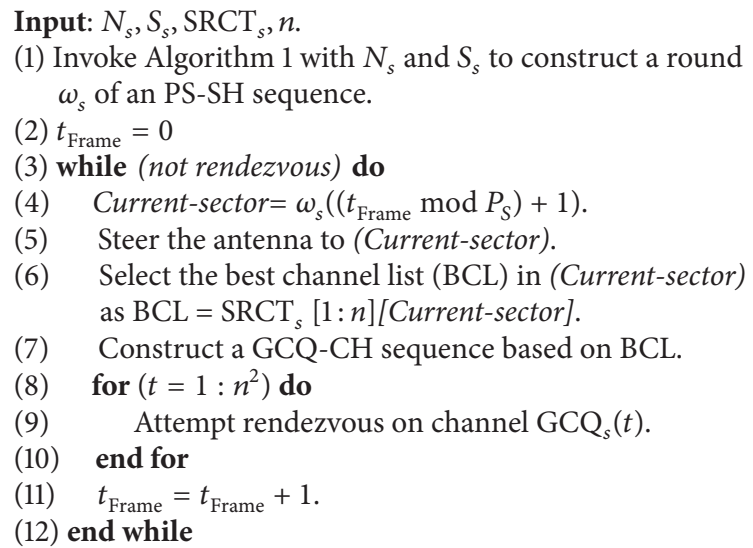

Algorithm 4: The sender SCH generation algorithm (PS-SCH).

Input: $N_{r}, S_{r}, \mathrm{SRCT}_{r}, n$.

(1) Invoke Algorithm 2 with $N_{r}$ and $S_{r}$ to construct a round $\gamma_{r}$ of an ES-SH sequence.

(2) $t_{\text {Frame }}=0$

(3) while (not rendezvous) do

(4) Current-sector $=\gamma_{r}\left(\left(t_{\text {Frame }} \bmod E_{r}\right)+1\right)$.

(5) Steer the antenna to (Current-sector).

(6) $\mathrm{BCL}=\mathrm{SRCT}_{s}[1: n]$ [Current-sector].

(7) Construct a GRQ-CH sequence based on BCL.

(8) for $\left(t=1: n^{2}\right)$ do

(9) Attempt rendezvous on channel $\mathrm{GRQ}_{r}(t)$.

(10) end for

(11) $t_{\text {Frame }}=t_{\text {Frame }}+1$.

(12) end while

Algorithm 5: The receiver SCH generation algorithm (ES-SCH). frame of the sender $\mathrm{SU}_{s}$ and a sector frame of its receiver $\mathrm{SU}_{r}$, a successful channel rendezvous is achieved between GCQ- $\mathrm{CH}_{s}$ and the GRQ- $\mathrm{CH}_{r}$. The channel rendezvous is guaranteed as long as there is a common channel between their $n$ best channels, where they can set up a link through the exchange of RTS/CTS messages.

For example, consider the pair of sender $\mathrm{SU}_{X}$ and receiver $\mathrm{SU}_{Y}$ in our previous example in Figure 1(a). The $\mathrm{SCH}$ sequences constructions for the pair of SUs and the rendezvous among them when they construct their combined $\mathrm{CH}$ sequences based on $n=2$ best-quality channels are illustrated in Figure 9. As a sender, $\mathrm{SU}_{X}$ in Figure 9(a) combines each sector of its PS-SH sequence with a grid column (GCQ-CH) sequence based on the two best-ranked channels of the respected column for the sector in $\mathrm{SRCT}_{X}$. For instance, the sector $S_{1}$ is combined with a GCQ-CH sequence generated based on the two best channels $\{2,4\}$

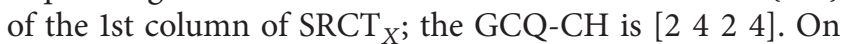
the other hand, each ES sector of the ES-SH sequence for the receiver $\mathrm{SU}_{Y}$ in Figure $9(\mathrm{~b})$ is combined with a grid row (GRQ-CH) sequence based on the best two channels of the $\mathrm{SRCT}_{Y}$ column which corresponds to the sector number. For instance, $\mathrm{SU}_{Y}$ combine the GRQ-CH sequence $\left[\begin{array}{llll}6 & 6 & 8 & 8\end{array}\right]$ within its sector $S_{1}$ which is constructed based on the best channels $\{6,8\}$. The sector and channel rendezvous between the SUs when they start their PES-SCH sequences synchronously is shown in Figure 9(c). The rendezvous is achieved during the 25th and 28th time slots on the sector rendezvous pair $\left(S_{2}\right.$ of $\mathrm{SU}_{X}, S_{3}$ of $\left.\mathrm{SU}_{Y}\right)$, as well as on the rendezvous channels $\{1,6\}$. However, when $S_{X}$ starts its $\mathrm{SCH} 4$ time slots later than $\mathrm{SU}_{Y}$, the channel and sector rendezvous is achieved after 5 time slots only from the $\mathrm{SCH}$ start time of $\mathrm{SU}_{X}$.

5.3.2. Combined IPES-SCH Scheme. For this symmetric role scheme, any $\mathrm{SU}$ can generate its $\mathrm{SCH}$ sequence according 


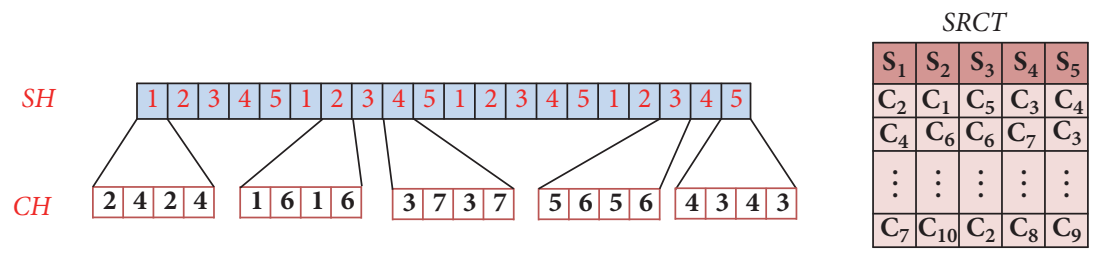

$\mathrm{SCH}$

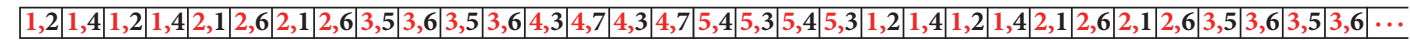

(a) The combined PES-SCH sequence construction for the sender $\mathrm{SU}_{X}$

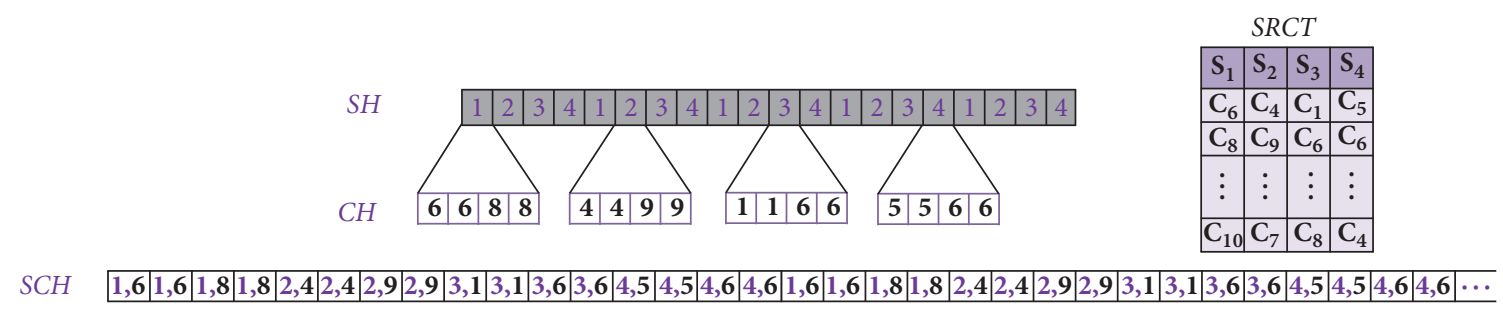

(b) The combined PES-SCH sequence construction for the receiver $\mathrm{SU}_{Y}$

\begin{tabular}{l|l|l|l|l|l|l|l|l|l|l|l|l|l|l|l|l|l|l|l|l|l|l|l|l|l|l|l|l|l|l|l|l|l|l|l|l|l|l|l|l|}
\hline Time slot & 0 & 1 & 2 & 3 & 4 & 5 & 6 & 7 & 8 & 9 & 10 & 11 & 12 & 13 & 14 & 15 & 16 & 17 & 18 & 19 & 20 & 21 & 22 & 23 & 24 & 25 & 26 & 27 & 28 & 29 & 30 & 31 & 32 & 33 & 34 & 35 & 36 & 37 & 38 & 39 \\
\hline
\end{tabular}

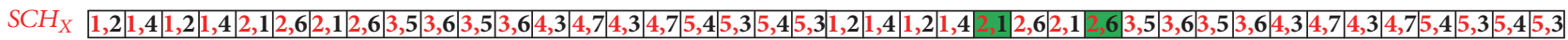

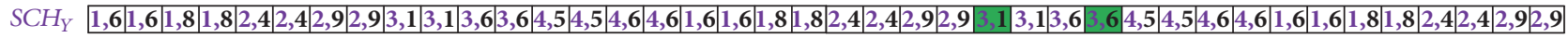

(c) Synchronous sector and channel rendezvous between $\mathrm{SU}_{X}$ and $\mathrm{SU}_{Y}$

\begin{tabular}{|c|c|}
\hline Time slot & \begin{tabular}{|l|l|l|l|l|l|l|l|l|l|l|l|l|l|l|l|l|l|l|l|l|l|l|l|l|l|l|l|l|l|l|l|l|l|l|l|}
0 & 1 & 2 & 3 & 4 & 5 & 6 & 7 & 8 & 9 & 10 & 11 & 12 & 13 & 14 & 15 & 16 & 17 & 18 & 19 & 20 & 21 & 22 & 23 & 24 & 25 & 26 & 27 & 28 & 29 & 30 & 31 & 32 & 33 & 34 & 35 \\
\end{tabular} \\
\hline $\mathrm{SCH}_{X}$ & $4,7|4,3| 4,7|5,4| 5,3 \mid 5,4[5,3|1,2| 1,4|1,2| 1,4|2,1| 2,6$ \\
\hline
\end{tabular}

(d) Asynchronous sector and channel rendezvous between the $\mathrm{SU}_{\mathrm{s}}$ when $\mathrm{SU}_{Y}$ starts its hopping earlier than $\mathrm{SU}_{X}$ by 4 slots

Figure 9: Rendezvous between SUs $X$ and $Y$ according to the combined PES-SCH scheme. $N_{X}=5$ and $N_{Y}=4$. Number of best-quality channels per each sector $n=2$.

to Algorithm 6. During each sector frame of its interleaved $\mathrm{SH}$ sequence that is constructed using the method in Section 4.1.2, the SU executes one type of the AGQ-CH sequences according to the type of the frame. Specifically, if the sector frame is a prime-based (P-frame), the SU generates a GCQ-CH based on the $n$ best-quality channels in the sector. On the other hand, when the sector is an even-based (Eframe), the SU generates a GRQ-CH. This will ensure that whenever the sector rendezvous occurs between a P-frame of $\mathrm{IPES}_{i}$ and an E-frame of $\mathrm{IPES}_{j}$, channel rendezvous is achieved between the $\mathrm{GCQ}_{i} \mathrm{CH}$ sequence and the $\mathrm{GRQ}_{j} \mathrm{CH}$ sequence, and vice versa.

5.4. Performance Analysis of the Combined SCH Schemes. In this subsection, we analyze the theoretical performance for the combined SCH schemes. Specifically, we prove the guaranteed rendezvous between any two SUs performing the combined SCH scheme by deriving the maximum time-torendezvous (MTTR).

Theorem 8. For any scheme $\mathbb{S}$ of our $\mathrm{SH}$ schemes which is combined with the AGQ-CH scheme for $n$ best-quality channels, the MTTR of the combined SCH is (MSRL $(\mathbb{S}) \times$ $\left.n^{2}\right)$. MSRL (S) here denotes the maximum sector rendezvous latency for the $\mathbb{S} S H$ scheme.
Input: $N_{i}, S_{i}$, Extended-ID a, $\mathrm{SRCT}_{i}, n$.

(1) Construct an IPES-SH sequence $U_{i}$ as explained in Section 4.1.2.

(2) period $=\left(P_{i} \times E_{i} \times\right.$ length $\left.(\mathbf{a})\right)$.

(3) $t_{\text {Frame }}=0$

(4) while (not rendezvous) do

(5) Current-sector $=U_{i}\left(\left(t_{\text {Frame }}\right.\right.$ mod period $\left.)+1\right)$.

(6) Steer the antenna to (Current-sector).

(7) $\mathrm{BCL}=\mathrm{SRCT}_{i}[1: n]$ [Current-sector].

(8) if (Current-sector is a P-sector) then

(9) Construct a GCQ-CH sequence based on BCL.

(10) for $\left(t=1: n^{2}\right)$ do

(11) Attempt rendezvous on channel $\mathrm{GCQ}_{i}(t)$.

(12) end for

(13) else

(14) Construct a GRQ-CH sequence based on BCL.

(15) for $\left(t=1: n^{2}\right)$ do

(16) Attempt rendezvous on channel $\mathrm{GRQ}_{i}(t)$.

(17) end for

(18) end if

(19) $t_{\text {Frame }}=t_{\text {Frame }}+1$.

(20) end while

Algorithm 6: IPES-SCH generation algorithm. 
Proof. To prove that the combined PES-SCH has MTTR = $\left(P_{i} E_{j}\right) \times n^{2}$, it suffices to prove that the pair of combined PES$\mathrm{SCH}$ sequences, for example, $\mathrm{SCH}_{i}$ and $\mathrm{SCH}_{j}$, can achieve successful sector and channel rendezvous within $\left(P_{i} E_{j}\right) \times n^{2}$ time slots.

Without loss of generality, assume that $\mathrm{SU}_{j}$ starts its $\mathrm{SCH}$ sequence $\delta$ time slots earlier than the start of $\mathrm{SU}_{i}$. In each $\mathrm{SCH}$ period of $\mathrm{SU}_{i}$, the $\mathrm{SCH}$ sequence of $\mathrm{SU}_{j}$ is rotate $\left(\mathrm{SCH}_{j}, \delta\right)$. As we stated before, the $\mathrm{SCH}$ sequence can be viewed as a series of sector frames which are composed of $n^{2}$ channel time slots. Let the clock drift $\delta=n^{2} \times \delta_{F}+\delta_{S}$, where $\delta_{F} \in$ $\left[0, E_{j}-1\right]$ denotes the shift amount of the sector frames and $\delta_{S} \in\left[0,\left(n^{2}\right)-1\right]$ denotes the shift of the channel time slots. Next, we prove the theorem in the following two cases.

Case $1\left(\delta_{S}=0\right)$. This case implies that the sector frames are perfectly aligned. In this case, since the SUs stay for similar number of time slots in each sector which is $\left(n^{2}\right)$ for executing their corresponding AGQ-CH sequences, for any value of $\delta_{F}$, there must exist an entire overlap between a PSframe of $\mathrm{SCH}_{i}$ and an ES-frame of $\mathrm{SCH}_{j}$ where the sector rendezvous happened. By Theorem 1, we know that the sector rendezvous is guaranteed to occur between a PS sector frame $h_{i}$ and an ES sector frame $h_{j}$ within $\left(P_{i} E_{j}\right)$ sector frames. As a consequence, the channel rendezvous is guaranteed to happen between the $\mathrm{GCQ}_{i} \mathrm{CH}$ sequence of $h_{i}$ and the $\mathrm{GRQ}_{j}$ $\mathrm{CH}$ sequence of $h_{j}$. Thus, the MTTR is $\left(P_{i} E_{j}\right)$ multiplied by the sector frame length $n^{2}$. Figures $9(\mathrm{c})$ and $9(\mathrm{~d})$ show the sector and channel rendezvous between $\mathrm{SU}_{X}$ and $\mathrm{SU}_{Y}$ when $\delta_{S}=0$ for two different values of $\delta_{F}$ and for $n=2$. In Figure 9(c), when $\delta_{F}=0$ and hence the clock drift $\delta=$ $\left(n^{2} \times 0+0\right)=0, \mathrm{SU}_{X}$ can achieve the sector rendezvous with $\mathrm{SU}_{Y}$ on the second round of its $\mathrm{SH}$ frames. The SUs rendezvous on their commonly available channels (i.e., $\mathrm{CH}_{1}$ and $\left.\mathrm{Ch}_{6}\right)$. However, when $\delta_{F}=1$ and $\delta=\left(n^{2} \times 1+0\right)=4$, $\mathrm{SU}_{X}$ can rendezvous with $\mathrm{SU}_{Y}$ earlier where it rendezvous with $\mathrm{SU}_{Y}$ during the first round of its $\mathrm{SH}$ frames.

Case $2\left(\delta_{S} \neq 0\right)$. When there is a time slot shift $\delta_{S} \in\left[1, n^{2}\right]$, it implies that the sector frames are not aligned. For any value of $\delta_{F}$, it is easily verified that each PS sector frame of $\mathrm{SCH}_{i}$ is overlapped with partial channel time slots form two consecutive ES frames of $\mathrm{SCH}_{j}$. Specifically, the PS frame of $\mathrm{SCH}_{i}$ overlaps with $n^{2}-\delta_{S}$ time slots from the former ES frame and $\delta_{S}$ time slots from the later ES frame of $\mathrm{SCH}_{j}$. Recall the proof of Theorem 1 , when $\mathrm{SU}_{i}$ repeats each round of its sector frames for $E_{r}$ times, each occurrence of the $h_{i}$ sector frame must overlap with different sector frame from the $E_{r}$ sector frames of $\mathrm{SU}_{j}$. The sector rendezvous is achieved when $h_{i}$ overlaps with $h_{j}$. However, considering the time slot shift $\delta_{S}$ of the $\mathrm{SCH}_{j}$ sequence, we can prove in the same way as case 1 that, within the same rendezvous delay bound $\left(\left(P_{i} E_{j}\right) \times n^{2}\right.$ time slots), there must exist at least two occurrences of $h_{i}$ which overlaps with different partial time slots of $h_{j}$. In one of these occurrences, the overlap happens between the first $n^{2}-\delta_{S}$ time slots of $h_{i}$ and the last $n^{2}-\delta_{S}$ time slots of $h_{j}$. On the other occurrence, the overlap happens between the last $\delta_{S}$ time slots of $h_{i}$ and the first $\delta_{S}$ time slots of $h_{j}$. Accordingly, channel rendezvous can be achieved in any of these two partial sector rendezvous chances where partial slots of the $\mathrm{GCQ}_{i} \mathrm{CH}$ sequence in $h_{i}$ overlap with other partial slots from the GRQ $\mathrm{CH}_{j}$ sequence in $h_{j}$. For example, Figure 10 illustrates the asynchronous rendezvous between $\mathrm{SU}_{X}$ and $\mathrm{SU}_{Y}$ when $\delta_{F}=0$ for three different values of $\delta_{S}$.

Summarizing the discussion above, we show that, under the combined PES-SCH, any two SUs are guaranteed to achieve channel rendezvous within $\left(\left(P_{i} E_{j}\right) \times n^{2}\right) \mathrm{SCH}$ period. We highlight here that the theoretical MTTR for the combined IPES-SCH is $\left(2 m \times \max \left\{\left(P_{i} E_{j}\right),\left(E_{i} P_{j}\right)\right\}\right) \times n^{2}$. It can be proven in a similar way to the proof of the PES-SCH MTTR by considering the two alignment cases of the sector frames.

\section{Results and Discussions}

In this section, we evaluate the performance of our developed directional antenna rendezvous schemes and compare them with three related works in $[19,28,30]$. The former two selected schemes for comparisons are the only schemes in the literature which tackle the rendezvous issue in DIR-CRNs. However, since both of them are asymmetric-role, we select the third scheme [30] which is proposed for rendezvous in the $60 \mathrm{GHz}$ networks for the comparison with our symmetricrole IPES scheme. This scheme follows a similar ID-based approach to our IPES-SH scheme. However, the extended ID in this scheme is relatively longer and the adjusted parameters of the number of sectors which are used for constructing the interleaved $\mathrm{SH}$ sequences are different.

6.1. Performance Comparison of the SH Schemes. We first compare the performance of our $\mathrm{SH}$ schemes with the two blind $\mathrm{SH}$ schemes in $[28,30]$. The $\mathrm{SH}$ scheme in [19] is not compared here because it is not a blind scheme. Extensive simulation experiments are conducted to compute the sector rendezvous latency (SRL) between two neighboring SUs (a sender $\mathrm{SU}_{i}$ and a receiver $\mathrm{SU}_{j}$ ). In the simulations, we consider different antenna configurations for the SUs where we simulate several combinations for the number of sectors $\left(N_{i}\right.$ and $\left.N_{j}\right)$. The relative locations of $\mathrm{SU}_{i}$ and $\mathrm{SU}_{j}$ represented by $\left(h_{i}, h_{j}\right)$ as well as the clock drift are randomly generated. For the ID-based schemes, two different 7-bit IDs are randomly assigned to the SUs. For each $\left(N_{i}\right.$ and $N_{j}$ ) combination, the simulation results are obtained by conducting $10^{5}$ independent runs where we accordingly compute the average and maximum SRL (ASRL and MSRL).

Figure 11 depicts the SRL results for the asymmetricrole SH schemes (our PES-SH and Li and Xie's SH [28]). The results illustrate the significant reduction on the SRL provided by our PES-SH in terms of ASRL and MSRL under all the different antenna configurations of the pair of SUs. For some antenna configurations, PES-SH can reduce the ASRL and MSRL significantly up to $80 \%$ and $84 \%$, respectively. The shorter ASRL and MSRL provided by the PES-SH scheme are due to its efficient design which relies on the coprimality between the adjusted prime and even numbers of $N_{i}$ and $N_{j}$, respectively. And since $N_{i}$ is prime for all the sender 


\begin{tabular}{|c|c|}
\hline Time slot & 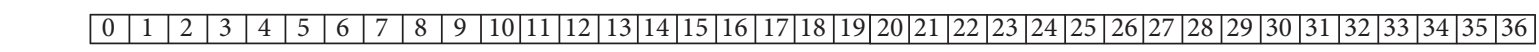 \\
\hline & 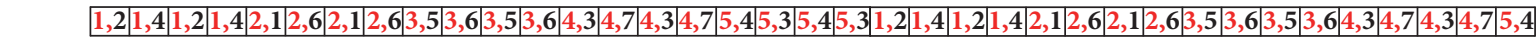 \\
\hline$Y, 1$ & 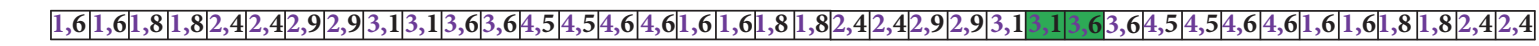 \\
\hline & \begin{tabular}{|l|l|l|l|l|l|l|l|l|l|l|}
$3,6,6$ & 4,5 & 4,5 & 4,6 & 4,6 & 1,6 & 1,6 & 1,8 & 1,8 & 2,4 \\
\end{tabular} \\
\hline
\end{tabular}

FIGURE 10: Asynchronous rendezvous between $\mathrm{SU}_{X}$ and $\mathrm{SU}_{Y}$ according to the combined PES-SCH scheme for $n=2$ best-ranked channels. $\mathrm{SCH}_{Y}$ is started earlier than $\mathrm{SCH}_{X}$ with $\delta_{S} \in[1,3]$.

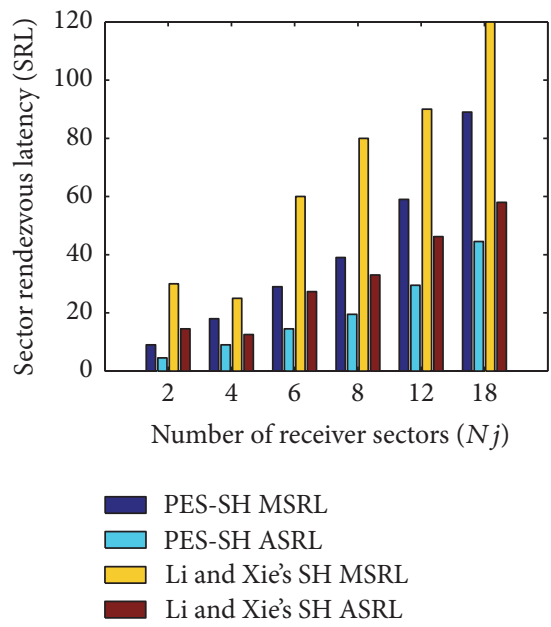

(a) $N_{i}=5$

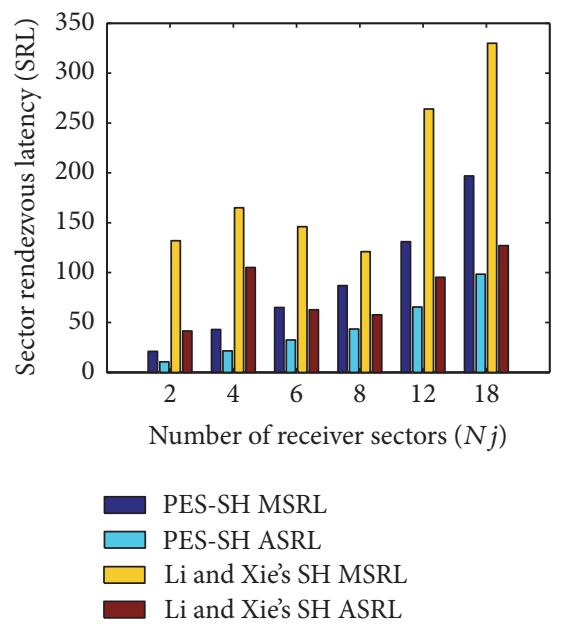

(b) $N_{i}=11$
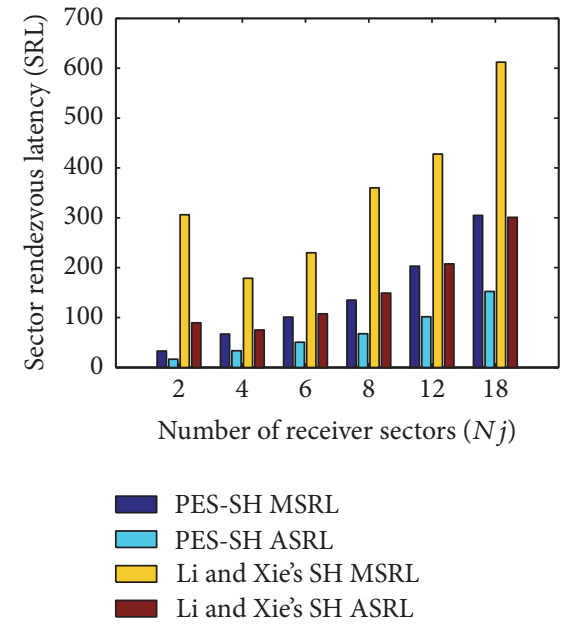

(c) $N_{i}=17$

FIGURE 11: Comparison of the sector rendezvous latency between our PES-SH scheme and Li and Xie's SH scheme [28] under fixed $N_{i}$ and varying $N_{j}$.

$\mathrm{SU}_{i}$ antenna configurations while the values of $N_{j}$ are even numbers that are valid for use (i.e., not in the AES set), this allows the sender $\mathrm{SU}_{i}$ in our PES-SH to achieve sector rendezvous with its receiver $\mathrm{SU}_{j}$ within $N_{i} N_{j} \mathrm{SH}$ period.

On the other side, the design of Li and Xie's SH scheme requires $N_{i}$ and $N_{j}$ to be adjusted to primes. Furthermore, the sender should perform an initiating round-robin $\mathrm{SH}$ sequence with a length of $N_{i}^{2}$ before it converts to the regular prime-based sequence. Accordingly, this will prolong the $\mathrm{SH}$ period needed for achieving the sector rendezvous to $\left(N_{i}^{2}+\right.$ $N_{i} P_{j}$ ) which actually represents the theoretical upper bound for the MSRL of Li and Xie's SH scheme when $\left(N_{i} \neq P_{j}\right)$. We can notice that this upper bound is tight for some $\left(N_{i}\right.$ and $N_{j}$ ) combinations, while it is not for others. Specifically, when $\left(N_{i} \% P_{j}=1\right)$ where $P_{j}$ is the adjusted prime for $N_{j}$, the sender $\mathrm{SU}_{i}$ cannot rendezvous with its receiver during its initiating $N_{i}^{2}$ round-robin $\mathrm{SH}$ sequence. However, when it starts following the regular prime-based $\mathrm{SH}$ sequence, the sector rendezvous happens within the subsequent $N_{i} P_{j}$ slots, which results in $\left(N_{i}^{2}+N_{i} P_{j}\right)$ MSRL. For example, when $N_{i}=$ 11 and $N_{j}=4$ in Figure 11(b), the sector rendezvous occurs in $(121+11 \times 5)=165$ sector slots. The same thing can be noticed for the cases when $N_{j}=2$ in Figures 11(a), 11(b), and 11(c), where the MSRL is $\left(N_{i}^{2}+N_{i} \times 2\right)$. This explains the irregular increased results in the figures for such $\left(N_{i}\right.$ and $\left.N_{j}\right)$ combinations.
In Figure 12, the SRL results for the symmetric-role $\mathrm{SH}$ schemes (our IPES-SH and the Chen et al.s SH scheme [30]) are shown. In the figure, it is shown that our IPES-SH scheme outperforms the other compared scheme especially in terms of MSRL. This improvement is due to the shorter SH period provided by IPES for achieving the sector rendezvous. In both schemes, the SH period for sector rendezvous depends mainly on the extended ID length as well as the adjusted numbers for $\left(N_{i}\right.$ and $\left.N_{j}\right)$ that are used for constructing the interleaved sequences. However, the adjusted parameters used by our IPES scheme are smaller than those used by the other scheme. Firstly, in the IPES-SH scheme, the extended ID length is $2 m=14$ which is relatively smaller than the $2 m+$ $1=15$ extended ID used by the other scheme. Furthermore, the interleaved SH sequences in Chen et al.s SH scheme are constructed by adjusting the number of sectors to a prime number as well as to a number that is a power-multiple of two (i.e., $q_{k}=2^{b_{k}}$, where $b_{k}$ is the smallest integer satisfying $2^{b_{k}} \geq N_{k}$ ). However, these adjusted numbers must be coprime with the extended ID length in order to achieve a successful sector rendezvous. Meanwhile, in IPES-SH, the interleaved PS and ES sequences are constructed based on the closest prime number and the closest even number which is not in the AES set. Hence, under most of the simulated antenna configurations, the adjusted $E_{i}$ and $E_{j}$ in IPES are smaller than the adjusted $q_{i}$ and $q_{j}$ parameters in the other scheme. 


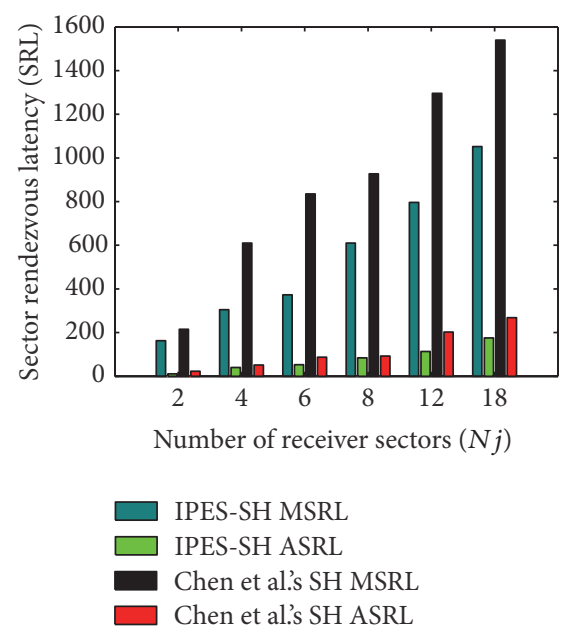

(a) $N_{i}=5$

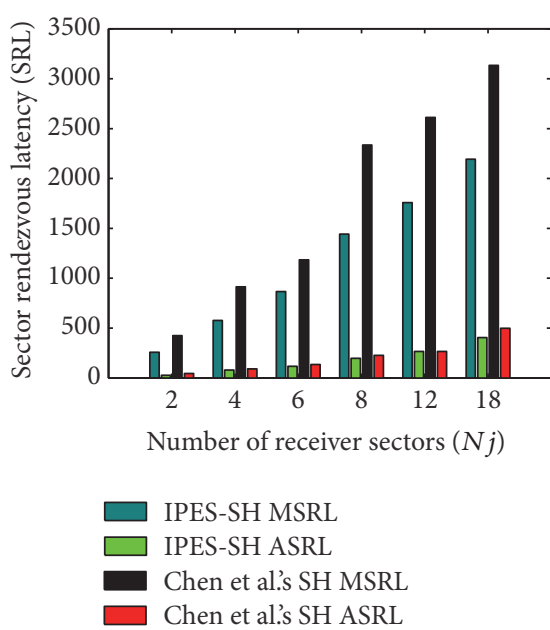

(b) $N_{i}=11$

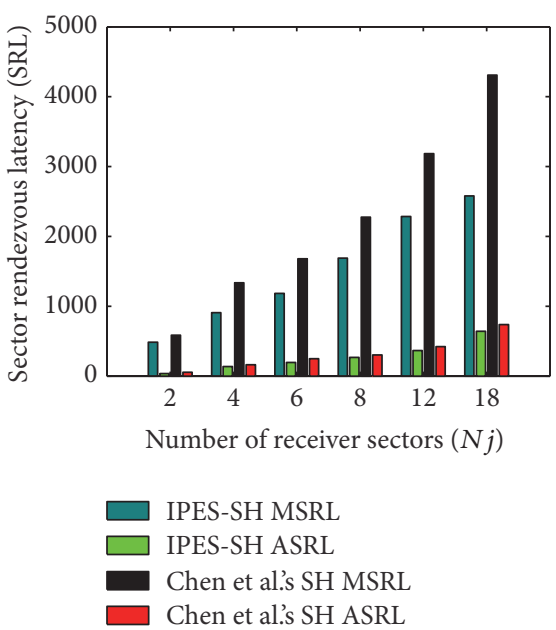

(c) $N_{i}=17$

FIGURE 12: Comparison of the sector rendezvous latency between our IPES-SH scheme and Chen et al.s SH scheme [30] under fixed $N_{i}$ and varying $N_{j}$. ID length $=7$ bits.

For example, in our IPES-SH scheme, $E_{j}=N_{j}$ for all the $N_{j}$ values, while the $q_{j}$ adjusted parameters for the $N_{j}$ values in Chen et al.'s SH scheme are $\{2,4,8,8,16,32\}$. Moreover, when $N_{i}$ or $N_{j}$ is $\leq 5$, the adjusted prime number used in IPES is 5 , while it is 7 on the other scheme because 7 is the closest prime that is coprime with the 15-bit extended ID. According to that, SUs in the IPES scheme can achieve the sector rendezvous faster than Chen et al.s SH scheme.

\subsection{Performance Comparison of the Combined SCH Schemes.} In this subsection, we compare the channel rendezvous performance of our SCH schemes with the other works under a practical DIR-CRN. The simulation configuration parameters are shown in Table 1.

We start our SCH simulation by comparing the performance of our combined PES-SCH with the combined $\mathrm{SCH}$ schemes of Song and Xie [19] and Li and Xie [28]. We evaluate our PES-SCH for $n=4$ best-quality channels which correspond to a AGQ-CH period of 16 time slots in each sector (i.e., the frame length $=16$ ). The other selected schemes for comparison are simulated with their proposed $\mathrm{CH}$ schemes. In [19], the SH scheme is combined with the asymmetric-role $\mathrm{CH}$ scheme in [35], where the sender SU stays for $L^{2}$ slots on each sector frame of its $\mathrm{SH}$ sequence performing a sequential fast $\mathrm{CH}$ on the $\left(C_{i} \subseteq L\right)$ available channels within the sector. Meanwhile, the receiver SU stays for $\left(N_{i}+1\right) \times L^{2}$ slots on each sector of its $N_{i}$ sectors performing a slow $\mathrm{CH}$ on all of its $\left(C_{j} \subseteq L\right)$ available channels. On the other side, the $\mathrm{SH}$ scheme of $\mathrm{Li}$ and Xie [28] is combined with the EJS-CH scheme [10]. However, due to the long period of the EJS-CH scheme required to guarantee channel rendezvous, $\mathrm{Li}$ and Xie simulated their SCH scheme by letting SUs stay for smaller period on each sector of their SH frames. During this period, SUs execute the corresponding subsequences of their generated EJS-CH sequence. Therefore, we simulate Li and Xie's SCH scheme
TABLE 1: Simulation parameters.

\begin{tabular}{lc}
\hline Network area deployment & $500 \mathrm{~m} \times 500 \mathrm{~m}$ \\
Number of channels $(L)$ & 10 \\
Number of PUs & 50 \\
The radius of the SU sensing/transmission range & $150 \mathrm{~m}$ \\
Slot duration for sending a packet on a channel & $2 \mathrm{~ms}$ \\
The PU packet arrival rate & 10 packets/s \\
The length of a PU packet & 120 slots \\
\hline
\end{tabular}

with a similar sector frame length to our PES-SCH scheme (i.e., 16 slots). All the schemes are simulated under different asynchronous settings where the clock drifts amount between SUs is selected randomly in each experiment.

Figure 13 depicts the TTR simulation results for the three schemes when they are evaluated under different antenna configurations for the pair of SUs. Even though the combined $\mathrm{SCH}$ scheme of Song and Xie is not a blind scheme, it produces very long TTR results. This is due to the large sector frame length where the sender and receiver stay for $L^{2}$ and $L^{2} \times\left(N_{i}+1\right)$ time slots, respectively, on each sector for performing their corresponding $\mathrm{CH}$ sequence. As a consequence, the $\mathrm{SCH}$ period needed for achieving rendezvous is $N_{j} \times\left(L^{2} \times\left(N_{i}+1\right)\right)$ which is very long as compared to the period of our PES-SCH scheme. On the other side, $\mathrm{Li}$ and Xie's SCH scheme can provide shorter ATTR than Song and Xie's scheme because it is simulated with smaller frame length. However, the MTTR of this scheme cannot be traced because we observe that TTR in some cases cannot be achieved within the whole simulation duration which is set to $5 \times 10^{4}$ slots. The reason for the indeterministic behaviour of $\mathrm{Li}$ and Xie's SCH scheme is because the stay period in each sector (i.e., 16 slots) is insufficient to guarantee rendezvous while using the EJS-CH scheme. According to the EJS-CH 

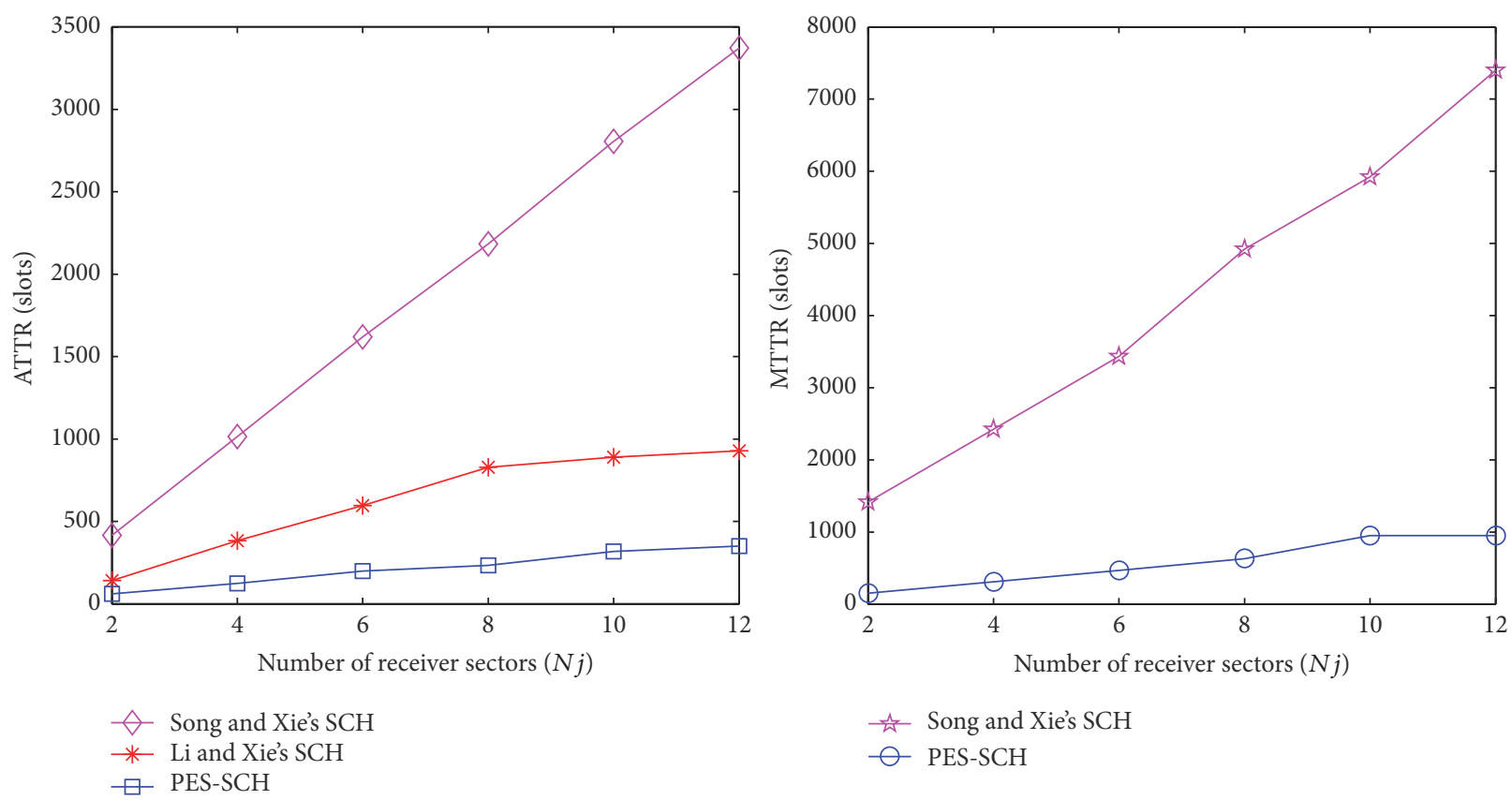

Figure 13: Comparison of the time-to-rendezvous (TTR) between the asymmetric-role SCH schemes when $N_{i}=5$.

[10], the rendezvous cannot be guaranteed unless the EJS$\mathrm{CH}$ sequences of the communicating SUs are overlapped for at least $4 P_{L}\left(P_{L}+1-G\right)=44(12-G)$ time slots. $P_{L}$ is the smallest prime number greater than $L$, while $G$ is the number of common available channels between the SUs available channels. Hence, to ensure rendezvous regardless of any misalignment of their local clocks, the SUs should stay for at least $4 P_{L}^{2}$ on each sector of their $\mathrm{SH}$ sequences to perform their EJS-CH sequences. This trade-off between the bounded MTTR of Song and Xie's SCH scheme and the small ATTR of Li and Xie's SCH scheme illustrates the efficiency of our proposed AGQ-CH scheme, which allows PES-SCH to guarantee rendezvous with the shortest TTR results.

In the second setting of the $\mathrm{SCH}$ simulation, we combine the $\mathrm{SH}$ schemes of $\mathrm{Li}$ and Xie [28] and Chen et al. [30] with our proposed AGQ-CH scheme. This is in order to evaluate their channel rendezvous performance when they are combined with an efficient and suitable $\mathrm{CH}$ scheme. Figures 14 and 15 show the TTR results of these schemes and our schemes when they are simulated for different values of the best-quality channel $n(2 \leqslant n \leqslant 5)$. In the simulation, we consider different values for the sender number of sectors $\left(N_{i}=\{4,6,12\}\right)$, while the receiver number of sectors $N_{j}$ is set as 4 . The figures show that our schemes always outperform the other schemes under all the different antenna configurations of the SUs. This is mainly due to the shorter SRL of our SH schemes and, as explained in Section 5.4, TTR depends on the SRL of the SH scheme coupled with the length of the sector frame $\left(n^{2}\right)$. Moreover, the figures illustrate the effect of $n$ (i.e., the number of best-quality channels) on the rendezvous performance where we can notice that as $n$ increases, the TTR results for all the schemes increase.
This is because the larger $n$, the longer the frame length $n^{2}$. Accordingly, when SUs spend longer period in each sector, they may waste more time while attempting rendezvous on other sectors rather than the rendezvous sector. This will result in a longer TTR until they can achieve a successful sector and channel rendezvous.

Finally, to illustrate the performance gain brought by applying directional antennas for channel rendezvous, we compare the performance of our combined PES-SCH scheme with the AAsync- $\mathrm{CH}$ omnidirectional antenna rendezvous scheme [16]. The AAsync-CH is selected among the other omnidirectional $\mathrm{CH}$ schemes since it is designed for asymmetric-role environment (i.e., SUs have preassigned role) similar to our PES-SCH. The schemes are compared in terms of their probabilities for achieving a successful channel rendezvous under different number of PUs in the network area. In our PES-SCH, the simulated sender and receiver SUs have $N_{i}=5$ and $N_{j}=6$ number of sectors and the number of best-quality channels $n=3$.

Figure 16 shows the significant outperformance of our directional antenna scheme as compared with the omnidirectional antenna scheme. It can be noticed from the figure that when the number of PUs in the network increases, the probability of the successful channel rendezvous for the omnidirectional scheme decreases dramatically. Meanwhile, in the PES-SCH, this probability is almost 1 for most of the cases and only decreases slightly when the number of PUs is close to 100 . This is due to the smaller interference region of the directional antenna, which results in higher channel availability for the SUs as compared to the omnidirectional antenna. So, when the total number of PUs in the network area increases, only few of them may reside within 

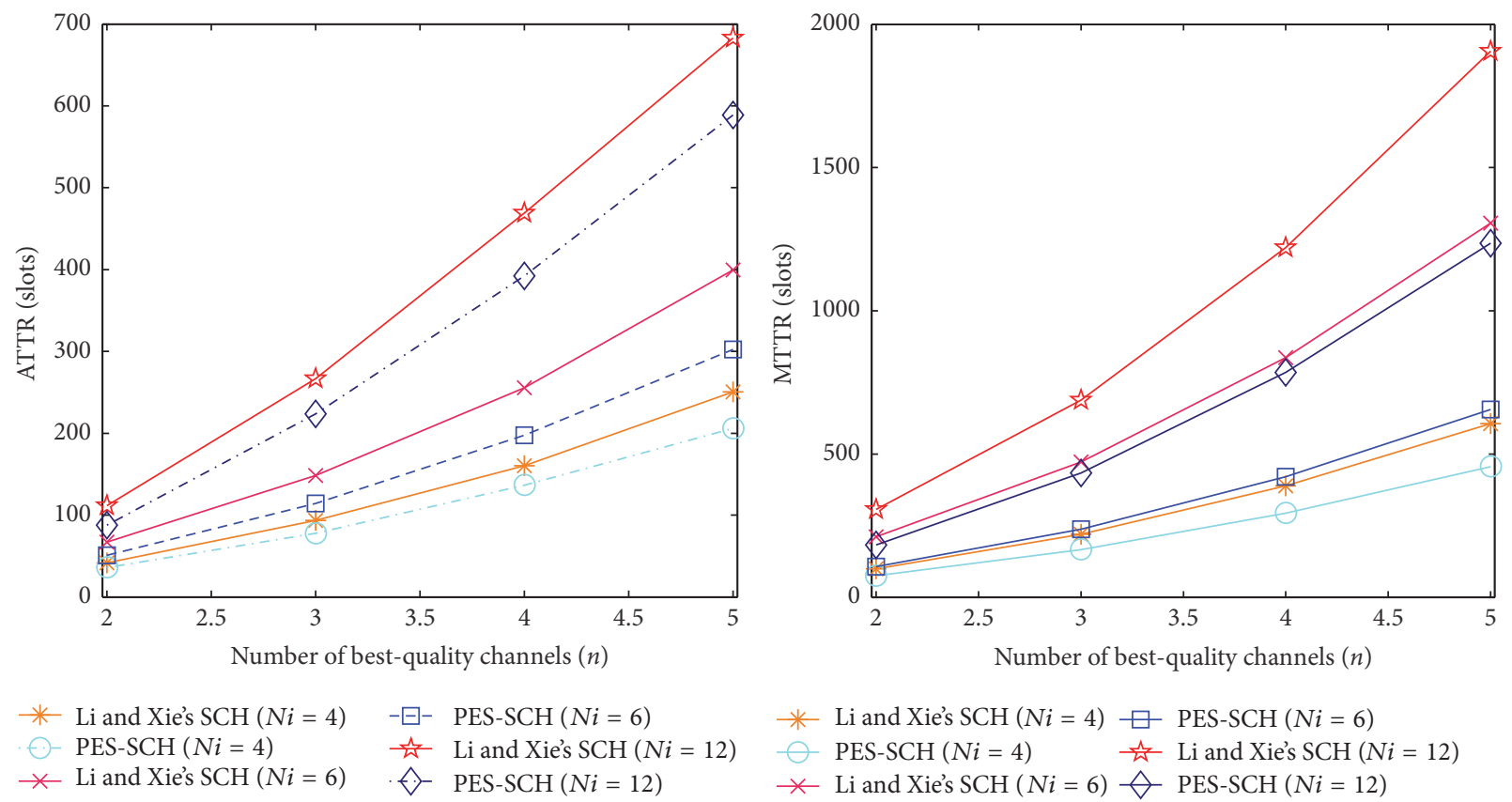

FIgURE 14: Comparison of the time-to-rendezvous (TTR) between the asymmetric-role SCH schemes when $N_{j}=4$.
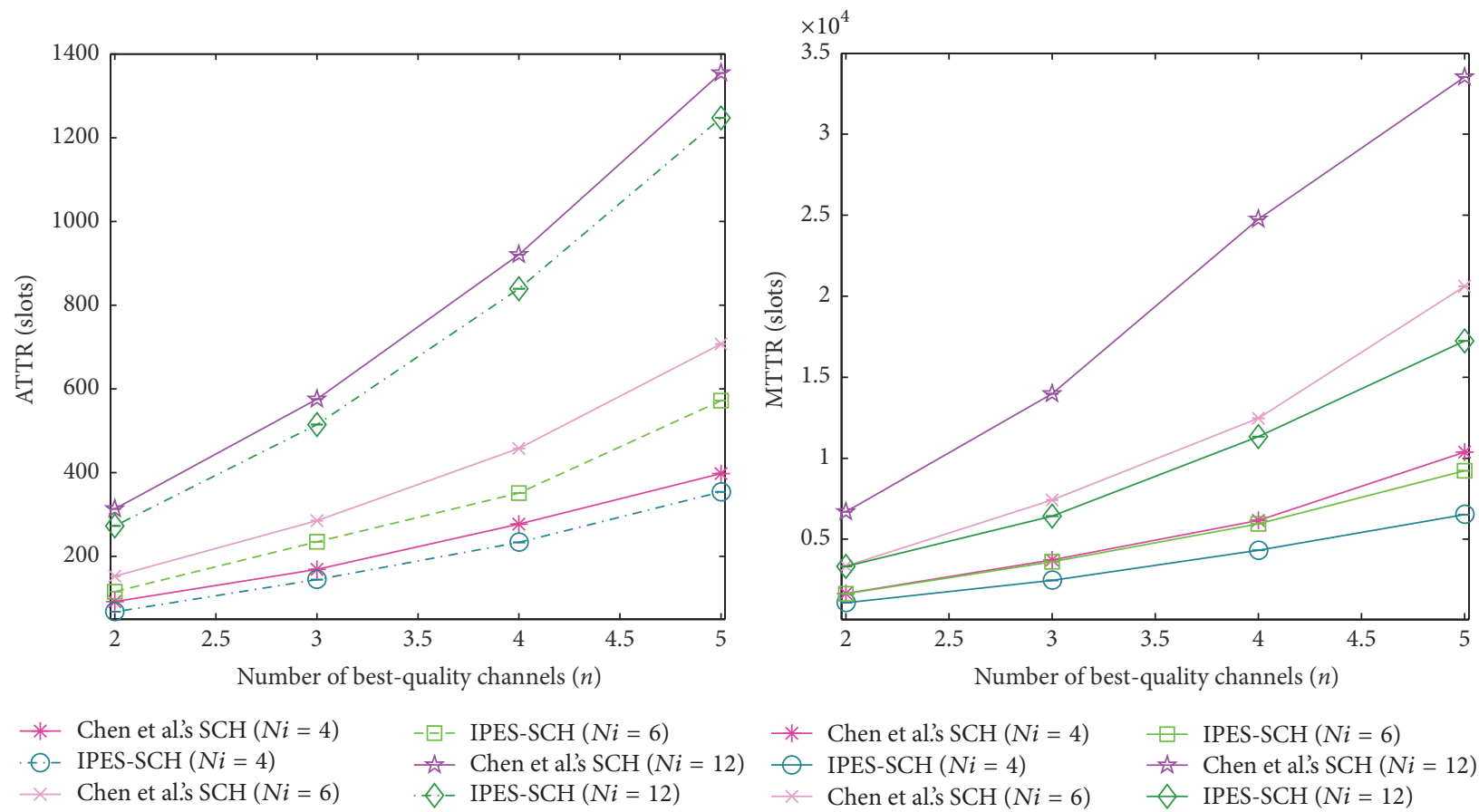

$$
\begin{aligned}
& -\square-\text { IPES-SCH }(\mathrm{Ni}=6) \\
& - \text { Chen et al.s SCH }(\mathrm{Ni}=12) \\
& \diamond-\text { IPES-SCH }(\mathrm{Ni}=12)
\end{aligned}
$$

$-\mathrm{IPES}-\mathrm{SCH}(\mathrm{Ni}=4)$
$\times \quad$ Chen et al.s SCH $(\mathrm{Ni}=6)$

FIGURE 15: Comparison of the TTR between the symmetric-role SCH schemes when $N_{j}=4$. ID length $=7$ bits.

the SUs rendezvous sectors where they can only impose a slight change in the channel availability. Accordingly, the probability of having common channels between the SUs on their sector rendezvous pair is always high, which increases the probability of successful channel rendezvous.
These results justify the motivation of our proposed schemes where equipping SUs with directional antennas can significantly enhance the channel rendezvous performance in high-density PU networks as compared with the omnidirectional antennas paradigm. 


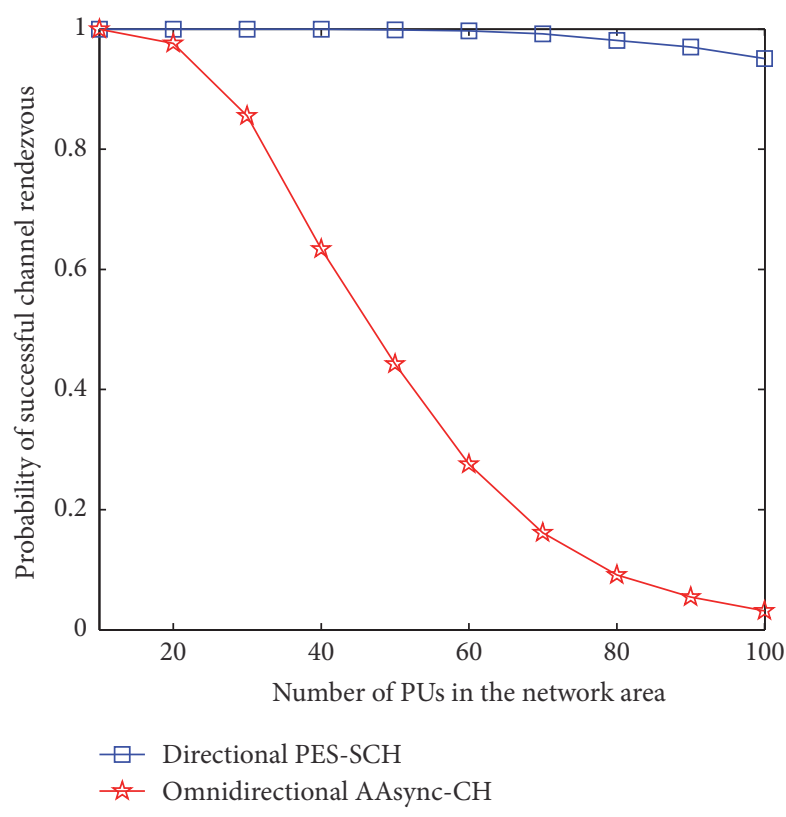

FIGURE 16: Comparison of the successful channel rendezvous probability between our PES-SCH directional antenna scheme and the AAsync-CH omnidirectional scheme.

\section{Conclusions}

In this paper, we studied the sector and channel rendezvous problem for SUs in DIR-CRNs. Two efficient coprimalitybased sector hopping schemes have been proposed for providing sector rendezvous in asymmetric- and symmetricrole environments. The $\mathrm{SH}$ schemes are combined with an efficient grid-quorum-based $\mathrm{CH}$ scheme for providing a guaranteed sector and channel rendezvous simultaneously between the SUs in DIR-CRNs. Theoretical analysis and extensive simulations are conducted to demonstrate the efficiency of the proposed schemes. The simulations results verify that our schemes significantly outperform the previous directional antenna rendezvous schemes (in terms of SRL and TTR). Furthermore, they demonstrate that our directional antenna rendezvous paradigm is more resistant to rendezvous failures under high-density PU CRNs, as compared with the omnidirectional antenna rendezvous paradigm.

\section{Conflicts of Interest}

The authors declare that they have no conflicts of interest.

\section{Acknowledgments}

The authors would like to acknowledge Malaysian Ministry of Higher Education for funding this study under the Fundamental Research Grant Scheme (FRGS) entitled "On the Cogitation of Primary User Reappearance in Cognitive Radio Network" (Project Code: MOHE: FRGS/1/2015/TK10/ UPM/02/2, UPM:03-01-15-1626FR, Vote no. 5524731).

\section{References}

[1] N. C. Theis, R. W. Thomas, and L. A. DaSilva, "Rendezvous for cognitive radios," IEEE Transactions on Mobile Computing, vol. 10, no. 2, pp. 216-227, 2011.

[2] B. F. Lo, "A survey of common control channel design in cognitive radio networks," Physical Communication, vol. 4, no. 1, pp. 26-39, 2011.

[3] M.-R. Kim and S.-J. Yoo, "Distributed coordination protocol for ad hoc cognitive radio networks," Journal of Communications and Networks, vol. 14, no. 1, pp. 51-62, 2012.

[4] P. Ren, Y. Wang, and Q. Du, "CAD-MAC: a channel-aggregation diversity based MAC protocol for spectrum and energy efficient cognitive Ad Hoc networks," IEEE Journal on Selected Areas in Communications, vol. 32, no. 2, pp. 237-250, 2014.

[5] G. Prasad Joshi, S. Y. Nam, S. W. Kim, and B.-S. Kim, "Adaptive window size-based medium access control protocol for cognitive radio wireless sensor networks," Journal of Sensors, vol. 2016, Article ID 2049859, 9 pages, 2016.

[6] S. Omar, O. E. Ghandour, and A. M. A. El-Haleem, "Multipath activity based routing protocol for mobile cognitive radio Ad Hoc networks," Wireless Communications and Mobile Computing, vol. 2017, Article ID 5380525, 12 pages, 2017.

[7] A. Mahmud, Y. Lee, and I. Koo, "A fully distributed resource allocation mechanism for CRNS without using a common control channel," Mathematical Problems in Engineering, vol. 2015, Article ID 537078, 9 pages, 2015.

[8] G. P. Joshi, S. Y. Nam, and S. W. Kim, "Rendezvous issues in ad hoc cognitive radio networks," KSII Transactions on Internet and Information Systems, vol. 8, no. 11, pp. 3655-3673, 2014.

[9] S. Romaszko, "A rendezvous protocol with the heterogeneous spectrum availability analysis for cognitive radio ad hoc networks," Journal of Electrical and Computer Engineering, vol. 2013, Article ID 715816, 18 pages, 2013.

[10] Z. Lin, H. Liu, X. Chu, and Y.-W. Leung, "Enhanced jumpstay rendezvous algorithm for cognitive radio networks," IEEE Communications Letters, vol. 17, no. 9, pp. 1742-1745, 2013.

[11] J. Kim, G. Park, and K. Han, "A Rendezvous Scheme for SelfOrganizing Cognitive Radio Sensor Networks," International Journal of Distributed Sensor Networks, vol. 10, no. 1, article 315874, 2014.

[12] E. O. Guerra, V. A. Reguera, R. D. Souza, E. G. Fernández, and M. E. Pellenz, "Systematic construction of common channel hopping rendezvous strategies in cognitive radio networks," EURASIP Journal on Wireless Communications and Networking, vol. 2015, no. 1, 2015.

[13] G.-Y. Chang, J.-F. Huang, and Y.-S. Wang, "Matrix-based channel hopping algorithms for cognitive radio networks," IEEE Transactions on Wireless Communications, vol. 14, no. 5, pp. 2755-2768, 2015.

[14] B. Yang, W. Liang, M. Zheng, and Y.-C. Liang, "Fully distributed channel-hopping algorithms for rendezvous setup in cognitive multi-radio networks," IEEE Transactions on Vehicular Technology, vol. PP, no. 99, 2015.

[15] J.-P. Sheu, C.-W. Su, and G.-Y. Chang, "Asynchronous quorumbased blind rendezvous schemes for cognitive radio networks," IEEE Transactions on Communications, vol. 64, no. 3, pp. 918930, 2016.

[16] P. K. Sahoo and D. Sahoo, "Sequence-based channel hopping algorithms for dynamic spectrum sharing in cognitive radio networks," IEEE Journal on Selected Areas in Communications, vol. 34, no. 11, pp. 2814-2828, 2016. 
[17] W. Chen, G. Yang, M. Chang, and W. C. Kwong, "Construction and analysis of shift-invariant, asynchronous-symmetric channel-hopping sequences for cognitive radio networks," IEEE Transactions on Communications, vol. 65, no. 4, pp. 1494-1506, 2017.

[18] A. Al-Mqdashi, A. Sali, M. J. Abdel-Rahman, N. K. Noordin, S. J. Hashim, and R. Nordin, "Efficient rendezvous schemes for fast-varying cognitive radio ad hoc networks," Transactions on Emerging Telecommunications Technologies, article e3217, 2017. http://dx.doi.org/10.1002/ett.3217.

[19] Y. Song and J. Xie, "Enhancing channel rendezvous in cognitive radio networks with directional antennas," in Proceedings of the IEEE International Conference on Communications, ICC 2015, pp. 3702-3707, June 2015.

[20] H. Jung and I.-H. Lee, "Connectivity Analysis of MillimeterWave Device-to-Device Networks with Blockage," International Journal of Antennas and Propagation, vol. 2016, Article ID 7939671, 9 pages, 2016.

[21] A. M. Al-Samman, T. A. Rahman, M. H. Azmi et al., "Statistical modelling and characterization of experimental mmwave indoor channels for future $5 \mathrm{G}$ wireless communication networks," PLoS ONE, vol. 11, no. 9, pp. 1-29, 2016.

[22] Y. Zhao, Z. Wei, Y. Liu, Q. Zhang, and Z. Feng, "AngleDomain Spectrum Holes Analysis with Directional Antenna in Cognitive Radio Network," in Proceedings of the 2017 IEEE Wireless Communications and Networking Conference (WCNC), pp. 1-6, San Francisco, CA, USA, March 2017.

[23] H. Yazdani and A. Vosoughi, "On cognitive radio systems with directional antennas and imperfect spectrum sensing," in Proceedings of the 2017 IEEE International Conference on Acoustics, Speech and Signal Processing (ICASSP), pp. 35893593, New Orleans, LA, USA, March 2017.

[24] Y. Dai and J. Wu, "Boundary helps: Efficient routing protocol using directional antennas in cognitive radio networks," in Proceedings of the 10th IEEE International Conference on Mobile Ad-Hoc and Sensor Systems, MASS 2013, pp. 502-510, October 2013.

[25] S. Anamalamudi, M. Jin, and J. M. Kim, "Hybrid CCC based AODV routing protocol for cognitive radio ad-hoc networks with directional antennas," in Proceedings of the 7th International Conference on Ubiquitous and Future Networks, ICUFN 2015, pp. 40-45, July 2015.

[26] Y. Wang, Q. Wang, H.-N. Dai, H. Wang, Z. Zheng, and J. $\mathrm{Li}$, "On local connectivity of cognitive radio ad hoc networks with directional antennas," in Proceedings of the 2016 IEEE International Conference on Communication Systems, ICCS 2016, pp. 1-6, December 2016.

[27] L. T. Dung, T. D. Hieu, S.-G. Choi, B.-S. Kim, and B. An, "Impact of beamforming on the path connectivity in cognitive radio ad hoc networks," Sensors, vol. 17, no. 4, 2017.

[28] J. Li and J. Xie, "Directional antenna based distributed blind rendezvous in cognitive radio ad-hoc networks," in Proceedings of the 58th IEEE Global Communications Conference, GLOBECOM 2015, pp. 1-6, December 2015.

[29] C.-M. Chao, H.-Y. Fu, and L.-R. Zhang, "A fast rendezvousguarantee channel hopping protocol for cognitive radio networks," IEEE Transactions on Vehicular Technology, vol. 64, no. 12, pp. 5804-5816, 2015.

[30] L. Chen, Y. Li, and A. V. Vasilakos, "Oblivious neighbor discovery for wireless devices with directional antennas," in Proceedings of the 35th Annual IEEE International Conference on Computer Communications, IEEE INFOCOM 2016, pp. 1-9, April 2016.

[31] I. Niven, H. S. Zuckerman, and H. Montgomery, An Introduction to the Theory of Numbers, John Wiley \& Sons, New York, NY, USA, 1991.

[32] K. Bian and J.-M. J. Park, "Maximizing rendezvous diversity in rendezvous protocols for decentralized cognitive radio networks," IEEE Transactions on Mobile Computing, vol. 12, no. 7, pp. 1294-1307, 2013.

[33] M. J. Abdel-Rahman and M. Krunz, "Game-theoretic quorumbased frequency hopping for anti-jamming rendezvous in DSA networks," in Proceedings of the 2014 IEEE International Symposium on Dynamic Spectrum Access Networks, DYSPAN 2014, pp. 248-258, April 2014.

[34] A. Al-Mqdashi, A. Sali, N. K. Noordin, S. J. Hashim, R. Nordin, and M. J. Abdel-Rahman, "An efficient quorum-based rendezvous scheme for multi-radio cognitive radio networks," in Proceedings of the 2016 IEEE 3rd International Symposium on Telecommunication Technologies (ISTT), pp. 59-64, November 2016.

[35] Y. Song and J. Xie, "QB ${ }^{2}$ IC: A QoS-based broadcast protocol under blind information for multihop cognitive radio ad hoc networks," IEEE Transactions on Vehicular Technology, vol. 63, no. 3, pp. 1453-1466, 2014. 


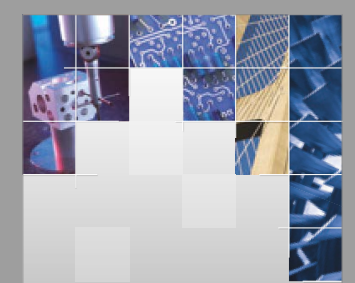

\section{Enfincering}
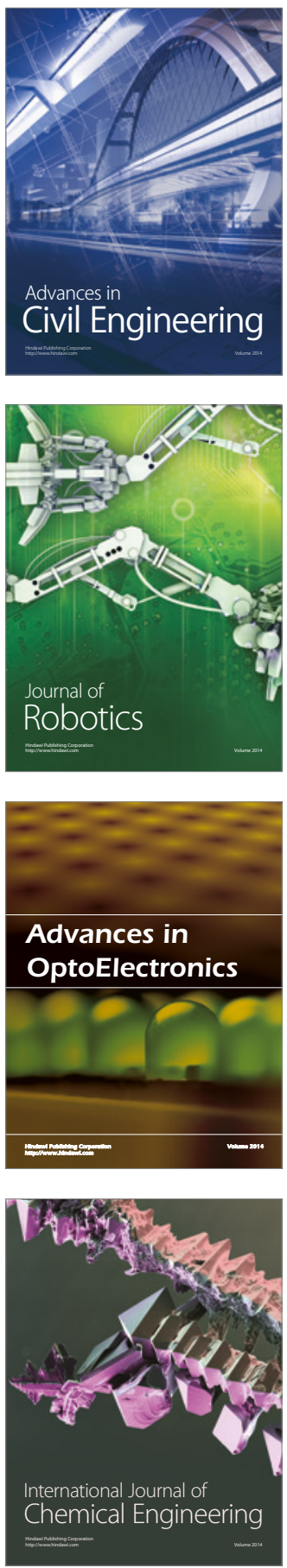

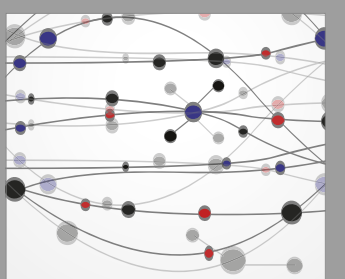

The Scientific World Journal

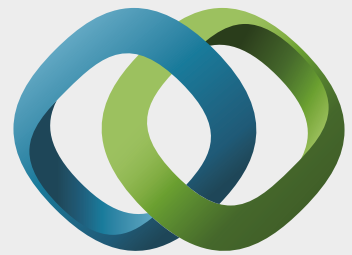

\section{Hindawi}

Submit your manuscripts at

https://www.hindawi.com
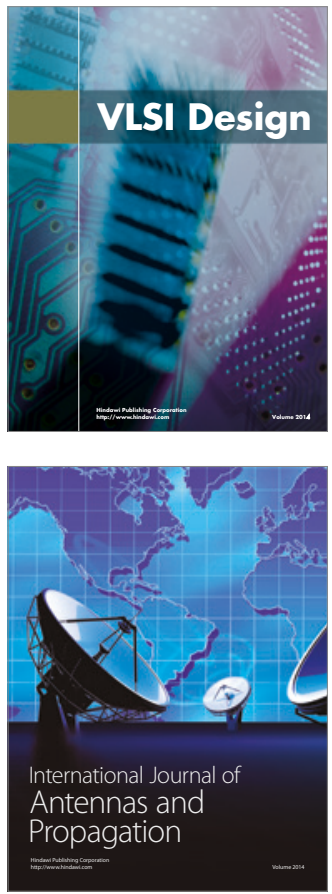

\section{Rotating}

Machinery
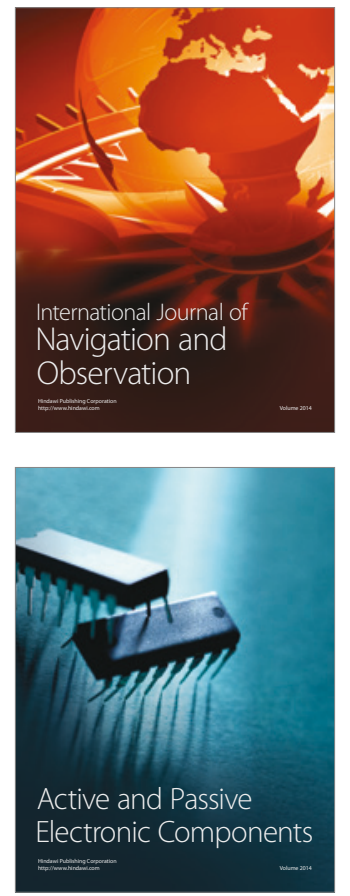
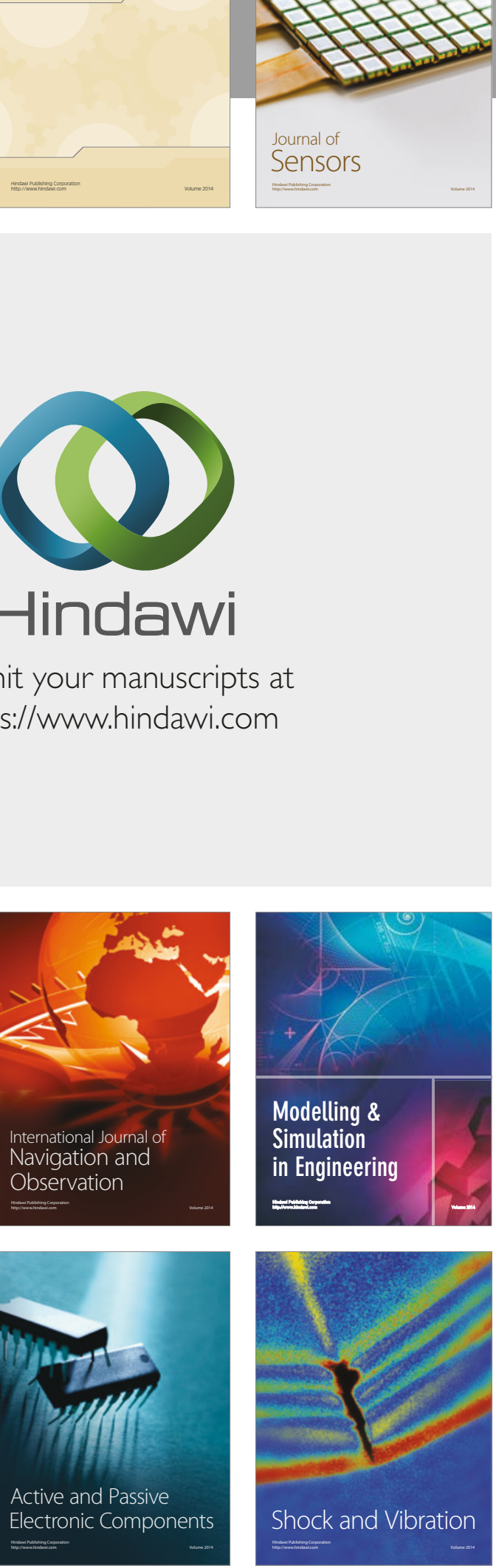
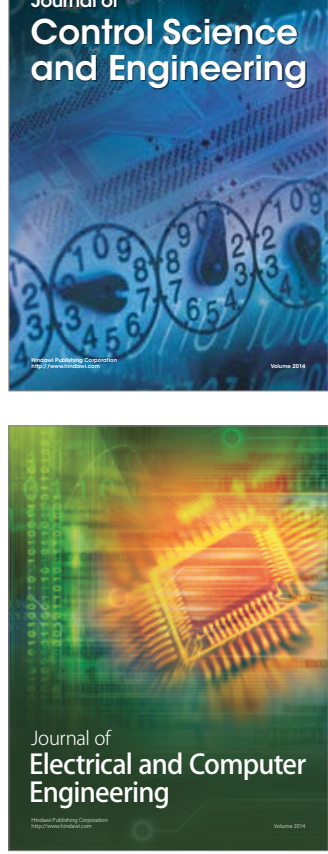

Distributed

Journal of

Control Science

and Engineering
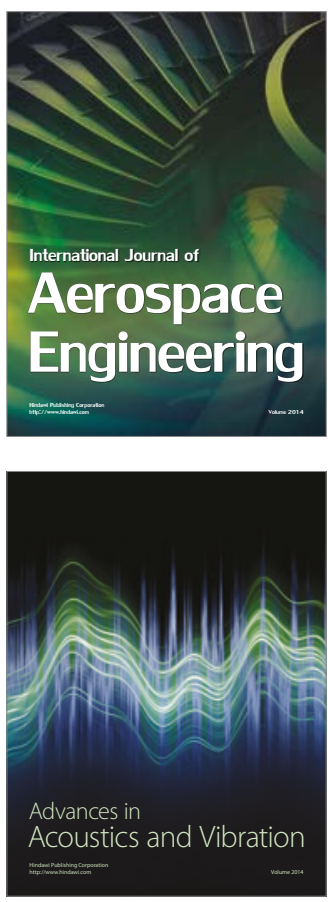

Sensor Networks 